\title{
A NOTION OF RECTIFIABILITY MODELED ON CARNOT GROUPS
}

\author{
SCOTT D. PAULS
}

\begin{abstract}
We introduce a notion of rectifiability modeled on Carnot groups. Precisely, we say that a subset $E$ of a Carnot group $M$ and $N$ is a $\operatorname{subgroup~of~} M$, we say $E$ is $N$ rectifiable if it is the Lipschitz image of a positive measure subset of $N$. First, we discuss the implications of $N$-rectifiability, where $N$ is a Carnot group (not merely a subgroup of a Carnot group), which include $N$-approximability and the existence of approximate tangent cones isometric to $N$ almost everywhere in $E$. Second, we prove that, under a stronger condition concerning the existence of approximate tangent cones isomorphic to $N$ almost everywhere in a set $E$, that $E$ is $N$-rectifiable. Third, we investigate the rectifiability properties of level sets of $C_{N}^{1}$ functions, $f: N \rightarrow \mathbb{R}$, where $N$ is a Carnot group. We show that for almost every $t \in \mathbb{R}$ and almost every noncharacteristic $x \in$ $f^{-1}(t)$, there exists a subgroup $T_{x}$ of $H$ and $r>0$ so that $f^{-1}(t) \cap B_{H}(x, r)$ is $T_{x^{-}}$ approximable at $x$ and an approximate tangent cone isomorphic to $T_{x}$ at $x$.
\end{abstract}

\section{INTRODUCTION}

The notion of rectifiability is central to the study of geometric measure theory, allowing for the proof of classical geometric properties in a much more general setting. In recent years, there has been significant interest and progress in the study of rectifiable sets not only in Euclidean space but in more general metric spaces as well (see, for example, Amb, AK99b, AK99a, Che99, DS97, FSSC95, FSSC99, GN96, Kir94, Mag01, Pan89, Whi98. Also, see the extensive bibliographies in GN96 and AK99b). In attempting to use the techniques of geometric measure theory to investigate the properties of general metric spaces, one quickly encounters a major difficulty: there may not be any rectifiable subsets or the set of rectifiable subsets may be too small to reveal any significant geometry. Here, we consider a subset of a metric space to be rectifiable if it can be realized as the Lipschitz image of a piece of Euclidean space. Thus, to have any hope of transporting the techniques of Euclidean geometric measure theory to metric spaces, we need a more general notion of rectifiable sets which are modeled on a wider class of metric spaces than simply Euclidean ones. In this paper, we investigate a special situation where much of the standard rectifiable theory carries over, but reveals some of the complications inherent in this endeavor.

We will restrict ourselves to the investigation of the so-called Carnot groups - connected, simply connected, graded nilpotent Lie groups equipped with a left-invariant CarnotCarathéodory metric (see below for precise definitions). Carnot groups arise in a variety of situations: in the asymptotic geometry of manifolds of negative curvature, in optimal

Key words and phrases. Carnot-Carathéodory metrics, rectifiability, stratified homogeneous spaces, Lipschitz maps, Carnot groups .

The author was partially supported by NSF grants DMS-9971563 and DMS-0306752. 
control theory, in the local geometry of equiregular Carnot-Carathéodory manifolds, in CR geometry, in the study of subelliptic partial differential equations, and in many other areas. In addition, Carnot groups are analytically very similar to Euclidean spaces (which are themselves particular examples of Carnot groups) in that they possess translations and dilations that respect the metric. Because of these similarities to Euclidean spaces, Carnot groups form a good test class for the generalization of the notion of rectifiability.

Further motivation for considering rectifiability modeled on Carnot groups arises in the study of the local geometry of Carnot-Carathéodory manifolds. A Carnot-Carathéodory manifold is a quadruple $\left(X, \mathcal{S},<\cdot, \cdot>, d_{X}\right)$ where $X$ is a smooth manifold, $\mathcal{S}$ is a subbundle of the tangent bundle, $\langle\cdot, \cdot\rangle$ is a smoothly varying inner product on the fibers of $\mathcal{S}$ and $d_{X}$ is the path metric formed by taking the infimum of lengths of paths (calculated with respect to $\langle\cdot, \cdot\rangle$ ) among absolutely continuous paths tangent a.e. to $\mathcal{S}$. Further, letting $\mathfrak{d}_{i}(x)$ be the dimension of the subspace of $T_{x} X$ spanned by all the commutators of order less than or equal to $i$, we say that $\left(X, \mathcal{S},<\cdot, \cdot>, d_{X}\right)$ is equiregular if the vector $\left(\mathfrak{d}_{0}(x), \mathfrak{d}_{1}(x), \ldots\right)$ is locally constant. Carnot groups are very special examples of equiregular Carnot-Carathéodory spaces given by the quadruple $\left(G, \mathcal{V},<\cdot, \cdot>, d_{G}\right)$ where $\mathcal{V}$ is the bottom level of the grading, thought of as a left invariant subbundle of $T G$, and $\langle\cdot, \cdot\rangle$ is a left invariant inner product on $\mathcal{V}$. For general equiregular Carnot-Carathéodory spaces, it is known (Mit85, see also Bel96]) that the tangent cone to $\left(X, \mathcal{S},<\cdot, \cdot>, d_{X}\right)$ is isometric to a Carnot group. However, the tangent group may vary from point to point (quite badly!) - see, for example, Bel96 and Var81. Naturally, this makes an investigation of the local geometry of Carnot-Carathéodory manifolds much more difficult than, for example, that of Riemannian manifolds. As we shall see, the framework of rectifiability modeled on Carnot groups identifies a subclass of CC manifolds which possess uniform local behavior.

Let $(M, d)$ be a Carnot group and let $N$ be a subgroup of $M$ equipped with a Carnot metric $d^{\prime}$. We define the following generalization of rectifiability: a subset $S \subset M$ is $N$-rectifiable if it is the Lipschitz (with respect to $d$ and $d^{\prime}$ ) image of a positive measure subset of $N$. This clearly generalizes the standard notion of rectifiability where $M$ and $N$ are replaced by Euclidean spaces of the appropriate dimension. Using this terminology, we will refer to the standard theory of rectifiability as $\mathbb{R}^{k}$-rectifiability. The main results of this paper concern the properties of $N$-rectifiable sets, recovering many of the basic facts about $\mathbb{R}^{k}$-rectifiable sets in $\mathbb{R}^{n}$ such as unique approximate tangent cones, approximative qualities of tangent cones. Precisely, we show the following theorems.

Theorem A. Let $N$ and $M$ be Carnot groups and suppose $E \subset M$ is a $N$-rectifiable set. Then,

- E is $N$-approximable

- For almost every $x \in E$, there exists a unique approximate tangent cone at $x$ which is isomorphic to $N$.

Roughly, a subset is $N$-approximable if at a.e. point there is a copy of $N$ sitting in $M$ which approximates $E$ locally in a measure theoretic sense. See definition 4.2 for a precise definition. An approximate tangent cone at $x \in E$ is a copy of $N=\exp _{x_{0}}(V)$ (for some base point $\left.x_{0}\right)$ in $M$ such that

$$
\frac{\mathscr{H}_{M}^{k}\left(E \cap B_{M}(x, r) \backslash X(x, V, s)\right)}{r^{k}} \rightarrow 0
$$


where $X(x, V, s)$ is roughly a "cone" over $N$ through a base point $x_{0}$ with "slope" $s$. See section 5 for the precise definition. Such notions are used in Mat95] in the case of $\mathbb{R}^{k}$ rectifiability. The reader should note that this theorem is restricted to $N$ which are full Carnot groups, not proper subgroups. As mentioned above, this theorem, among other applications, provides a class of Carnot-Carathéodory spaces which are quite well behaved (with respect to local geometry). The class of $N$-rectifiable submanifolds in a given Carnot group $M$ has the property that each member is a Carnot-Carathéodory manifold in its own right (by restriction of the distribution) and that the tangent cone at almost every point is isomorphic to $N$.

Mirroring the Euclidean situation, we would like to show that the rectifiability, approximability, and approximate tangent cone conditions are equivalent. Unfortunately, one cannot approach this via the standard arguments because the various projections onto copies of $N$ sitting in $M$ are almost never Lipschitz. However, if we define a strong approximate tangent cone at $x \in E$ to be a copy of $N$ satisfying two conditions:

(1)

$$
\frac{\mathscr{H}_{M}^{k}\left(E \cap B_{M}(x, r) \backslash X(x, V, s)\right)}{r^{k \cdot d e p t h(N)}} \rightarrow 0
$$

where $\operatorname{depth}(N)$ is the number of steps in the nilpotent Lie group $N$ and $\exp (V)=$ $N$

(2) For sufficiently small $s$ and $\varepsilon>0$,

$$
X(x, V, s) \cap X\left(x, V^{\perp}, s\right) \cap B_{M}(x, \varepsilon)=\emptyset
$$

Then we have the following theorem.

Theorem B. Suppose $E$ is a $\mathscr{H}_{M}^{k}$-measureable subset of a Carnot group $M$ and $V$ is a subspace of $\mathfrak{m}$ so that $\exp (V)$ is isomorphic to $N$. Further suppose that for $\mathscr{H}_{M}^{k}$ a.e. $y \in E$ $\exp (V)$ is a strong approximate tangent cone at $y$. Then, $E$ is $N$-rectifiable.

In Bel96, Bellaïche poses the following question: if a Carnot-Carathéodory manifold has a locally uniform tangent structure (i.e. if all tangent cones (in the sense of Gromov) in a neighborhood are isomorphic as Lie groups), then does there exist a Lipschitz map between the tangent cone at a point and a neighborhood of that point? Our notion of rectifiability addresses this question under the hypothesis that the Carnot-Carathéodory manifold is a submanifold of a Carnot group. Using theorem B, we see that if a submanifold of a Carnot group has strong approximate tangent cones isomorphic to $N$ almost everywhere (i.e. it has uniform tangent structures in this measure theoretic sense), then there is a local Lipschitz equivalence between $N$ and the Carnot-Carathéodory manifold.

Next, we turn to studying the rectifiability properties of submanifolds in Carnot groups that do not necessarily enjoy tangent structures which are Carnot groups themselves. We focus on a class of maps which appear in the work of Franchi, Serra Cassano and Serapioni ([FSSC99]), the $C_{N}^{1}$ maps. There are functions $f: N \rightarrow \mathbb{R}$ which have continuous horizontal derivatives (see the next section for a precise definiton).

Theorem C. Let $N$ be a Carnot group of Hausdorff dimension $k$ and let $f: N \rightarrow \mathbb{R}$ be a $C_{N}^{1}$ map. Then, for a.e. $t \in \mathbb{R}$ and $\mathscr{H}_{N}^{k-1}$ a.e. $x \in \operatorname{Int}\left(f^{-1}(t) \backslash \Sigma_{t}\right)$, there exists a subgroup $T_{x} \subset N$ and $r_{x}>0$ such that $B_{N}\left(x, r_{x}\right) \cap f^{-1}(t)$ is $T_{x}$-approximable at $x$ and $f^{-1}(t)$ has a unique approximate tangent cone isomorphic to $T_{x}$ at $x$. 
In the theorem, $\Sigma_{t}$ is the collection of characteristic points of the level set $f^{-1}(t)$ and $\operatorname{Int}\left(f^{-1}(t) \backslash \Sigma_{t}\right)$ denotes the interior of $f^{-1}(t) \backslash \Sigma_{t}$. We note that a priori, the interior may be empty. Note that in this theorem, we must allow $T$ to be a proper subgroup of $N$ and that $T$, equipped with the metric from $N$ restricted to $T$, may not be a Carnot group. This theorem is a step towards generalizing the techniques of geometric measure theory to the Carnot setting. In particular, this theorem gives information concerning generalizing the notion of slicing manifolds by Lipschitz maps. Unfortunately, as shown by the limitations in this theorem (and illustrated by an example in section 5) we cannot conclude that the level sets are $N^{\prime}$-rectifiable for some $N^{\prime}$ - we lack a Lipschitz map. However, as evidenced by the theorem, many of the approximative qualities of rectifiable sets are inherited by the level sets. This suggests a modification of the notion of rectifiable currents in the Carnot setting based on these types of properties.

The proofs of these theorems rest on extensions of Euclidean analytic tools to the Carnot case. The most useful one of these is the (suitably defined) differentiability of Lipschitz maps on Carnot groups which is originally due to Pansu (Pan89). The form of the theorem used in this paper is an extension due to Vodopyanov and Ukhlov (VU96) and recently proved using a different method by Magnani (Mag01). In addition, in sections 2 and 3, we prove various lemmas concerning the properties of the Hausdorff measure including a metric area formula. Most of the proofs of these lemmas are adaptations of arguments in Federer (Fed69] ) and the proof of the area formula follows Kirchheim's argument in Kir94. To prove theorems A and B, we follow arguments based on arguments in Mat95 extended using the lemmas and techniques described above. However, to prove theorem $\mathrm{C}$, we diverge from the classical arguments, instead using smooth approximations of the Lipschitz map (as in [FSSC95], [FSSC99] and [GN96]) and apply metric arguments akin to those in Pau98.

The structure of the paper is as follows: section 2 reviews some of the known measure theory and differentiability results for Carnot groups and proves many of the useful measure theoretic lemmas needed in the proofs, such as properties of Jacobians of maps and a weak Sard-like property. Section 3 is devoted to an area formula for Lipschitz maps between Carnot groups. Sections 4-6 introduce $N$-rectifiability, $N$-approximability and prove theorems A and B. Section 7 is devoted to proving theorem C.

\section{BACKGRound RESUlts FOR CARNOT GROUPS}

2.1. Distances and measures. Assume that $N$ is a connected, simply connected graded nilpotent Lie group. Recall that $N$ is graded if the Lie algebra decomposes as $\mathfrak{n}=\mathcal{V} \oplus$ $\mathcal{V}_{2} \oplus \ldots \oplus \mathcal{V}_{l}$ where $\left[\mathcal{V}_{1}, \mathcal{V}_{j}\right]=\mathcal{V}_{j+1}$

Definition 2.1. We will call the integer $l$ the depth of $N$ and denote it by

$$
\operatorname{depth}(N)
$$

We denote by $\mathcal{V}$ not only the bottom level of the grading, but the left invariant vector bundle generated by left translating $\mathcal{V}$ around $N$. We also assume that $\mathcal{V}$ is equipped with an inner product $\langle\cdot, \cdot\rangle$, which we also think of as a left invariant inner product on the subbundle $\mathcal{V}$. 
Definition 2.2. A Carnot group is a quadruple $\left(N, \mathcal{V},\langle\cdot, \cdot\rangle, d_{N}\right)$ where $N, \mathcal{V}$ and $\left.<\cdot, \cdot\right\rangle$ are as above. To define $d_{N}$ we let $\mathcal{H}$ be the absolutely continuous paths which are tangent almost everywhere to $\mathcal{V}$. Then, the Carnot-Carathéodory distance is defined as

$$
d_{N}\left(n_{1}, n_{2}\right)=\inf \left\{\int<\gamma^{\prime}, \gamma^{\prime}>^{\frac{1}{2}} \mid \gamma \in \mathcal{H} \text { and } \gamma \text { connects } n_{1} \text { to } n_{2}\right\}
$$

It follows from the construction that $d_{N}$ is a left invariant metric on $N$ which admits a homothety, denoted $h_{t}$. The homothety is defined by its action on the Lie algebra where it acts on vectors in $\mathcal{V}_{i}$ by multiplication by $t^{i}$.

We will often think of a Carnot group $N$ as identified with $\mathbb{R}^{k}$ via the exponential map and use an orthonormal basis $\left\{X_{i}\right\}$ for $\mathfrak{n}$, adapted to the grading, as our coordinates for $\mathbb{R}^{k}$. Given $n \in N$ and $r>0$, we define $\operatorname{Box}(n, r)=\left\{\exp _{n}\left(\left(t_{1}, \ldots, t_{k}\right)|| t_{j} \mid<r^{d(j)}\right\}\right.$ where $d(j)$ is the level of the grading to which $X_{j}$ belongs. Next, we recall the well known Ball-Box theorem (see for example, Gro96] or [Mon02]):

Theorem 2.3. Let $N$ be a Carnot group. Then $\exists C>0$ such that, given $n \in N$ and $r>0$,

$$
\operatorname{Box}\left(b, \frac{r}{C}\right) \subset B_{N}(n, r) \subset B o x(n, C r)
$$

While the definition of $d_{N}$ is geometrically compelling, it is very difficult to compute with. Luckily, one can use any of a family of quasi-norms on $N$ to aid in computation. We describe one here.

Definition 2.4. Suppose $N$ is a Carnot group with grading $\mathfrak{n}=\mathcal{V} \oplus \mathcal{V}_{2} \oplus \ldots \oplus \mathcal{V}_{l}$. For $n \in N$, decompose $n$ according to the Euclidean vector space basis for $\mathfrak{n}, n=e^{v_{1}+v_{2}+v_{3}+\ldots+v_{l}}$. Then, we define a quasi-norm on $N$ as follows:

$$
|n|_{q n}=\left(\sum_{i=1}^{\operatorname{depth}(N)}\left\|v_{i}\right\|^{\frac{2}{d\left(v_{i}\right)}}\right)^{\frac{1}{2}}
$$

where $d\left(v_{i}\right)$ is the level of the grading of which $v_{i}$ is a member and $\|\cdot\|$ is the Euclidean norm. We also define a function on $N \times N$ by $d_{q n}\left(n_{1}, n_{2}\right)=\left|n_{1}^{-1} n_{2}\right|_{q n}$.

Note that $d_{q n}$ is, by construction, left invariant and admits $h_{t}$ as a homothety. Thus, $d_{N}$ and $d_{q n}$ are biLipschitz equivalent. A good reference on quasi-norms on Lie groups is Goo76.

To any metric on a metric space, we can introduce an associated Hausdorff measure. To fix notation, we define the Hausdorff measures needed in this paper.

Given a Carnot groups $N$ with Carnot-Carathéodory metric $d_{N}$ and a subset $U$ of $N$. Let $\mathscr{E} \delta$ be the collection of closed $d_{N}$ balls of diameter less than or equal to $\delta$. Then,

$$
\mathscr{H}_{N, \delta}^{k}(U)=\inf \left\{\sum \operatorname{diam}_{N}\left(E_{i}\right)^{k} \mid U \subset \cup E_{i}, E_{i} \in \mathscr{E}_{\delta}\right\}
$$

And,

$$
\mathscr{H}_{N}^{k}(U)=\lim _{\delta \rightarrow 0} \mathscr{H}_{N, \delta}^{k}(U)
$$

Note that we do not use a normalization by, for example, the measure of the unit ball in $N$ and that we use covers by closed balls rather than covers by any sets. This Hausdorff measure is sometimes called the spherical Hausdorff measure on $N$. 
Definition 2.5. Let $N$ be a Carnot group. The Hausdorff dimension of a set $E \subset N$ is

$$
\sup \left\{s \mid \mathscr{H}_{N}^{s}(E)=0\right\}
$$

We quote a well known lemma (see, for example, Gro96] 0.6B):

Lemma 2.6. Let $S$ is a smooth hypersurface of a Carnot group $N$ where the Hausdorff dimension of $N$ is $k$. Then, the Hausdorff dimension of $S$ is $k-1$.

We next define the notion of Ahlfors regularity.

Definition 2.7. Let $X$ be a complete metric space and $\mu$ a Borel measure on $X$. We say $\mu$ is Ahlfors regular of dimension $s$ if there exists $C>0$ so that

$$
C^{-1} r^{s} \leq \mu\left(B_{X}(x, r)\right) \leq C r^{s}
$$

when $0<r \leq \operatorname{diam}(X)$ and $x$ is a point where $\mu\left(B_{X}(x, r)\right)>0$ for all $r>0$.

In this paper, we will be considering the Ahlfors regularity of $\mathscr{H}_{N}^{k-1}$ when restricted to $S$, a hypersurface of $N$ (where $N$ satisfies the assumption of the most recent lemma).

Because Carnot-Carathéodory metrics and quasi-norms on Carnot groups are left invariant, the Hausdorff measures associated to them are in fact all constant multiples of the Haar measure on such a group. Thus, many of the same measure theoretic results concerning densities, etc. are true for $\mathscr{H}_{N}^{k}$ that are true for Lebesgue measure in $\mathbb{R}^{m}$. For proofs and discussions of these facts, in a more general setting, see DS97. We will use the biLipschitz equivalence of $d_{N}$ and $d_{q n}$ and the relation between the Hausdorff measures freely in the computations below.

Lemma 2.8. Let $k$ be the Hausdorff dimension of $N$. If $U \subset N$ is $\mathscr{H}_{N}^{k}$ measurable then almost every point of $U$ is a Lebesgue density point. In other words, for almost every $a \in U$,

$$
\lim _{r \rightarrow 0} \frac{\mathscr{H}_{N}^{k}\left(U \cap B_{N}(a, r)\right)}{\mathscr{H}_{N}^{k}\left(B_{N}(a, r)\right)}=1
$$

Next, we define the usual densities.

Definition 2.9. If $N$ is a Carnot group and $U \subset N, x \in N$, we define

$$
\Theta_{N}^{*, k}(U, x)=\varlimsup_{\lim _{r \rightarrow 0^{+}}} \frac{\mathscr{H}_{N}^{k}\left(U \cap B_{N}(x, r)\right)}{r^{k}}
$$

and

$$
\Theta_{*, N}^{k}(U, x)=\underline{\lim }_{r \rightarrow 0^{+}} \frac{\mathscr{H}_{N}^{k}\left(U \cap B_{N}(x, r)\right)}{r^{k}}
$$

If both of these coincide, then the common value is denoted $\Theta_{N}^{k}(U, x)$.

Note that, due to the lack of normalization of the Carnot metrics and the related Hausdorff measures, our estimates will be much less precise than in the Euclidean case.

Lemma 2.10. If $U \subset N$ and $\mathscr{H}_{N}^{k}(U)<\infty$ then there exists a constant $C>0$, depending only on the structure of the group $N$ and the Carnot-Carathéodory metric on $N$ so that

(1) $C 2^{-k} \leq \Theta_{N}^{*, k}(U, x) \leq C$ for almost all $x \in U$.

(2) If $U$ is $\mathscr{H}_{N}^{k}$ measurable then $\Theta_{N}^{*, k}=0$ for almost all $x \in N \backslash U$. 
In this lemma (and in the rest of the paper), the phrase "depends on the structure of the group $N$ " means that the constant in question depends only on the dimensions of the levels of the grading of $\mathfrak{n}$. The proof of this theorem follows from the standard arguments in $\mathbb{R}^{n}$ (see e.g. Fed69, 2.10.18 or [Sim83, theorem 3.6) combined with the observation that the Hausdorff measure derived from any Carnot-Carathéodory metric on a Carnot group is left invariant and scales with the homothety.

Next we quote two useful formulae. Both can be found in Hei95 as equation 4.8 and proposition 4.9 respectively.

To state them, we assume $N$ is a Carnot group and pick a Riemannian completion of the inner product defining the Carnot-Carathéodory metric on $N$ which makes the grading orthogonal.

Definition 2.11. Let $N$ be a Carnot group and $\left\{X_{1}, \cdots, X_{a}\right\}$ be an orthonormal basis for $\mathcal{V}$. Let $\nabla f$ denote the Riemannian gradient of a function $f$ and denote by $\nabla_{0} f$ the horizontal gradient of $f$,

$$
\nabla_{0} f=X_{1} f X_{1}+\cdots+X_{a} f X_{a}
$$

Definition 2.12. Let $S$ be a smooth surface in $N$, a Carnot group. Suppose further that $S$ is a level set of some smooth function $f$. Then, $x \in S$ is called a characteristic point if $\nabla_{0} f(x)=0$.

Below $|\cdot|$ is the usual norm on $\mathbb{R}$.

Proposition 2.13 (Coarea formula). Suppose $f: N \rightarrow \mathbb{R}$ is a smooth map from a Carnot group of Hausdorff dimension $k$ to $\mathbb{R}$ and $u$ is any nonnegative measurable function. Then,

$$
\int_{N} u(x)\left|\nabla_{0} f(x)\right| d \mathscr{H}_{N}^{k}(x)=\int_{0}^{\infty} \int_{f^{-1}(t)} u(y) d \mathscr{H}_{N}^{k-1}(y) d t
$$

Proposition 2.14. Suppose $S$ is a level surface of a smooth real valued function $f$ on $N$, a Carnot group of Hausdorff dimension $k$. Then, for $\mathscr{H}_{N}^{k-1}$ almost everywhere on $S$,

$$
d \mathscr{H}_{N}^{k-1}=\frac{\left|\nabla_{0} f\right|}{|\nabla f|} d A
$$

where $d A$ is the Riemannian area element on $S$.

We note that the size of the characteristic locus has been investigated by many authors recently. In particular, Z. Balogh (Bal02 $)$ has shown that one can construct a $C^{1, \alpha}$ surface in the Heisenberg group, $\mathbb{H}$, with a characteristic locus of positive two-dimensional Euclidean Hausdorff measure. However, if the surface is at least $C^{1}$ then the characteristic locus has $\mathscr{H}_{N}^{k-1}$ measure zero. See, for example, Mag03 and Bal02.

We end this section with a definition of the $C_{N}^{1}$ functions.

Definition 2.15. If $N$ is a Carnot group and $f: N \rightarrow \mathbb{R}$ is a continuous function then $f \in C_{N}^{1}$ if $\nabla_{0} f$ exists (in the sense of distributions) and is a continuous vector valued function. 
2.2. Differentiability of Lipschitz maps. We first quote a definition and a result of P. Pansu (see [Pan89]) crucial to our constructions:

Definition 2.16. A map $f: N \rightarrow M$ between Carnot group is said to be differentiable in the sense of Pansu at $n \in N$ with differential $d f_{n}$ if the limit

$$
d f_{n}(y)=\lim _{s \rightarrow 0} h_{\frac{1}{s}}^{\prime} f(n)^{-1} f\left(n h_{s} y\right)
$$

exists and convergence is uniform for all $y \in N$.

The following theorem is a generalization of a theorem of Pansu (Pan89) due to Vodopyanov and Ukhlov (VU96]). Recently, Magnani (Mag01) has also proved this theorem using a different technique.

Theorem 2.17. If $N$ and $M$ are Carnot groups and $f$ is a Lipschitz map from a measurable set $U \subset N$ to $M$, then $f$ is differentiable almost everywhere (in the sense of Pansu) and the differential $d f_{x}$ is a graded group homomorphism at almost every point.

In [Pau98, the author proves a limited metric differentiability for Lipschitz maps of Carnot groups into complete metric spaces. In the special case when the target space is another Carnot group, either an extension of the author's arguments or an appeal to the result above gives complete metric differentiability. In some of the later results, it is more convenient to work with the metric version of differentiability, hence we state it here.

Theorem 2.18. Let $f: U \subset N \rightarrow M$ be a Lipschitz map between Carnot groups. Then $f$ is metrically differentiable almost everywhere. In other words, for almost every $n \in N$,

$$
\Delta_{n}\left(y_{1}, y_{2}\right)=\lim _{t \rightarrow 0}\left\{\frac{d_{M}\left(f\left(n^{\prime} h_{t} y_{1}\right), f\left(n^{\prime} h_{t} y_{2}\right)\right)}{t} \mid n^{\prime} \in B_{N}(n, t)\right\}
$$

exists, the limit converges uniformly and $\Delta_{n}$ admits a homothety and is left invariant under the action of $N$.

The following lemma dictates exactly how the image of the differential approximates the function at a point.

Lemma 2.19. Let $f: U \subset N \rightarrow M$ be a Lipschitz mapping between Carnot groups, where $U$ is a positive measure subset of $N$ and let $d f_{x}$ denote the Pansu differential at a point of differentiability, $x$. Then,

$$
d_{M}\left(f\left(x e^{v}\right), f(x) d f_{x}\left(e^{v}\right)\right)=o\left(d_{N}\left(e^{0}, e^{v}\right)\right)
$$

Proof: This is a straightforward computation. Let $v_{0}$ be the Lie algebra vector in the direction of $v$ such that $d_{N}\left(e^{0}, e^{v_{0}}\right)=1$ and let $t=d_{N}\left(e^{0}, e^{v}\right)$.

$$
\begin{aligned}
d_{M}\left(f\left(x e^{v}\right), f(x) d f_{x}\left(e^{v}\right)\right) & =d_{M}\left(f(x)^{-1} f\left(x h_{t} e^{v}\right), d f_{x}\left(h_{t} e^{v_{0}}\right)\right) \\
& =d_{M}\left(h_{t} d f_{x}\left(e^{v_{0}}\right), d f_{x}\left(h_{t} e^{v_{0}}\right)\right)+o(t) \\
& =o(t)
\end{aligned}
$$

The last equality stems from the fact that Pansu's differential intertwines the homotheties of $N$ and $M$. 
Remark: One should note that the resulting metric $\Delta_{x}$ is very close to being a CarnotCarathéodory metric on $N$ (the only possible degeneracy is that the inner product on each fiber may be only semi-definite). To see this, one combines the fact that the differential is a graded group homomorphism and the definition of the metric $\Delta_{x}$ with the previous lemma, concluding that one may recognize $\Delta_{x}\left(n_{1}, n_{2}\right)$ by the quantity $d_{M}\left(d f_{x}\left(n_{1}\right), d f_{x}\left(n_{2}\right)\right)$. As we shall see in the next section, at points of differentiability when the map is suitably nondegenerate, $\Delta_{x}$ is a well defined Carnot-Carathéodory metric.

2.3. Jacobians and a weak Sard-like theorem. Let $f: U \subset N \rightarrow M$ be a Lipschitz map between a positive measure subset of a Carnot group and another Carnot group and let $k$ be the Hausdorff dimension of $N$.

Definition 2.20. For each $x \in U \subset N$ we define the Jacobian of the map $f$ at $x$ by

$$
J(x)=\lim _{t \rightarrow 0}\left\{\frac{\mathscr{H}_{M}^{k}\left(f\left(B_{N}(y, t)\right)\right)}{\mathscr{H}_{N}^{k}\left(B_{N}(y, t)\right)} \mid y \in B_{N}(x, t)\right\}
$$

By arguments analogous to those in Pau98 concerning the existence of the metric differential, $J(x)$ exists almost everywhere and scales appropriately. Moreover, we now prove that the image of the set where $J(x)=0$ has $\mathscr{H}_{M}^{k}$-measure zero.

Lemma 2.21. Let $Z=\{x \in U \subset N \mid J(x)=0\}$. Then, $\mathscr{H}_{M}^{k}(f(Z))=0$.

Proof: Fix $\varepsilon>0, n_{0} \in N$ and $0<R<\infty$. Let $Z_{R}=Z \cap B_{N}\left(n_{0}, R\right)$. We will show that $f\left(Z_{R}\right)$ has measure zero. We may cover $Z_{R}$ by balls $B_{N}(n, r)$ with the property that $n \in Z_{R}$ and $\frac{\mathscr{H}_{M}^{k}\left(f\left(B_{N}(n, r)\right)\right)}{\mathscr{H}_{N}^{k}\left(B_{N}(n, r)\right)}<\varepsilon$. By the assumption that $J(n)=0$, this covering is fine, so using the Vitali covering lemma, we refine the cover to a countable disjoint collection of balls $\left\{B_{i}=B_{N}\left(n_{i}, r_{i}\right)\right\}$ with $r_{i}<R$ which cover almost all of $Z_{R}$. Since $f$ is Lipschitz, we know that $\cup_{i} f\left(B_{i}\right)$ covers $\mathscr{H}_{M}^{k}$ almost all of $f\left(Z_{R}\right)$ as well. Thus

$$
\begin{aligned}
\mathscr{H}_{M}^{k}\left(f\left(Z_{R}\right)\right) & \leq \sum_{i} \mathscr{H}_{M}^{k}\left(f\left(B_{N}\left(n_{i}, r_{i}\right)\right)\right) \\
& <\varepsilon \sum_{i} \mathscr{H}_{N}^{k}\left(B_{N}\left(n_{i}, r_{i}\right)\right) \\
& \leq \varepsilon \mathscr{H}_{N}^{k}\left(Z_{R}\right) \\
& \leq \varepsilon \mathscr{H}_{N}^{k}\left(B_{N}\left(n_{0}, R\right)\right)
\end{aligned}
$$

Thus, since $\varepsilon$ is arbitrary, $\mathscr{H}_{M}^{k}\left(f\left(Z_{R}\right)\right)=0$. The result now follows easily.

Next we prove a lemma analogous to Sard's theorem concerning the measure of the image of the "degenerate" set. Suppose $f: U \subset N \rightarrow M$ is a Lipschitz map and let $N_{x}=f(x) d f_{x}(N)$.

Lemma 2.22. Suppose $\operatorname{dim}_{\mathscr{H}}(N) \leq \operatorname{dim}_{\mathscr{H}}(M)$. Then, $\mathscr{H}_{M}^{k}\left(\left\{f(x) \mid \mathscr{H}_{M}^{k}\left(N_{x}\right)=0\right\}\right)=0$.

Proof: To begin with, we may assume that at all points Pansu's derivative exists and $J(x)$ exists for all $f(x)$ we are considering since the set of the complementary points has measure 
zero. First we observe that using the definition of Pansu's differential, the uniformity of its convergence, the left invariance and homothety of the Carnot-Carathéodory metric $d_{M}$, we have that $d_{M}\left(f\left(x h_{t} e^{v}\right), f(x) h_{t} d f_{x}\left(e^{v}\right)\right)=o(t)$. In particular, this says that $f\left(B_{N}(x, t)\right)$ lies in a $o(t)$ neighborhood of $f(x) d f_{x}\left(B_{N}\left(e^{0}, t\right)\right)$. Since $f(B)$ has Hausdorff dimension less than or equal to $k$, we know that $\mathscr{H}_{M}^{k}\left(f\left(B_{N}(x, t)\right)\right) \leq \mathscr{H}_{M}^{k}\left(d f_{x}\left(B_{N}(x, t)\right)\right)+o\left(t^{k}\right)$. Now consider an image point $f(x)$ such that $\mathscr{H}_{M}^{k}\left(N_{x}\right)=0$. We discuss separately two cases: $\operatorname{ker}\left(d f_{x}\right)=\left\{e^{0}\right\}$ and $\operatorname{ker}\left(d f_{x}\right) \neq\left\{e^{0}\right\}$. In the first case, this together with the fact that $d f_{x}$ is a graded group homomorphism implies that the image of $d f_{x}$ is isomorphic to $N$. Thus, $\Delta_{x}$ is positive definite on $N$ and, since $\Delta_{x}$ is left invariant and admits a homothety, $d f_{x}:\left(N, \Delta_{x}\right) \rightarrow\left(M, d_{M}\right)$ is biLipschitz onto its image and hence cannot have $\mathscr{H}_{M}^{k}$-measure zero. So, we may assume that the kernel is nontrivial. Hence, $N_{x}$ is isomorphic to a quotient of $N$ (by the kernel), call it $N^{\prime}$. In particular, realizing $N^{\prime}$ as a subgroup of $N$, we see that $\mathscr{H}_{N}^{k}\left(N^{\prime}\right)=0$ and so, since $d f_{x}$ is Lipschitz, $\mathscr{H}_{M}^{k}\left(N_{x}\right) \leq L^{k} \mathscr{H}_{N}^{k}\left(N^{\prime}\right)=0$. Thus, we have that $\mathscr{H}_{N}^{k}\left(f\left(B_{n}(x, t)\right)\right)=o\left(t^{k}\right)$, yielding the desired result.

\section{An AREA FORMUla}

In this section, we provide a proof of an area formula for Lipschitz maps. A change of variables formula is proved by Vodopyanov and Uhklov (VU96) using methods based on Pansu's techniques in Pan89]. One should be able to extend their arguments to prove the statement below. Also, recently, Magnani (Mag01) independently proved the same area formula using a different (but equivalent) definition of Jacobian. We include the proof here for completeness. Again, let $f: U \subset N \rightarrow M$ be a Lipschitz map of a Carnot group $N$ to a Carnot group $M$. Let $k$ be the Hausdorff dimension of $N$.

Lemma 3.1. Fix $\lambda>1$ and let $E$ be a measurable set in $N$ such that for every $x \in E, \Delta_{x}$ exists and is nondegenerate. Then there exists a countable Borel cover of $E,\left\{B_{i}\right\}$, and for each $B_{i}$, a left invariant $C C$ metric, $d_{i}$, on $N$ such that for all $x, y \in B_{i}$,

$$
\lambda^{-1} d_{i}(x, y) \leq d_{M}(f(x), f(y)) \leq \lambda d_{i}(x, y)
$$

Moreover,

$$
\lambda^{-k} \frac{\mathscr{H}_{i}^{k}\left(B_{N}(x, 1)\right)}{\mathscr{H}_{N}^{k}\left(B_{N}(x, 1)\right)} \leq J(x) \leq \lambda^{k} \frac{\mathscr{H}_{i}^{k}\left(B_{N}(x, 1)\right)}{\mathscr{H}_{N}^{k}\left(B_{N}(x, 1)\right)}
$$

for all $x \in B_{i}$ which are density points of $B_{i}$ with respect to the Hausdorff measure, $\mathscr{H}_{i}^{k}$, constructed with respect to the distance $d_{i}$.

Proof: This theorem follows exactly as lemma 4 in Kir94 which, in turn, follows much of the argument of lemma 3.2.2 in Fed69. The metrics $d_{i}$ are given by $d_{i}\left(n_{1}, n_{2}\right)=$ $d_{M}\left(d f_{x}\left(n_{1}\right), d f_{x}\left(n_{2}\right)\right)$.

While we explore the concept of $N$-rectifiability in detail in the next section, we define a set $E \subset M$ to be $N$-rectifiable if it is the Lipschitz image of a positive measure subset of $N$.

Corollary 3.2. If $E \subset M$ is $N$-rectifiable, then for almost every $x \in E, \Theta^{k}\left(\mathscr{H}_{M}^{k}\lfloor E, x)=\right.$ $\mathscr{H}_{N}^{k}\left(B_{N}\left(e^{0}, 1\right)\right)$. 
Proof: Using lemma 3.1 above, this follows at density points of the $B_{i}$. More precisely, fix $\lambda>1$ and let $B_{i}$ and $d_{i}$ be as in the lemma. Now, for each point of density of $B_{i}$ which is also a point of Pansu differentiability, let $K_{i}$ be the preimage of $B_{i}$. We have,

$$
\begin{aligned}
\lambda^{-2 k} \frac{\mathscr{H}_{N}^{k}\left(K_{i} \cap B_{N}\left(x, \frac{\delta}{\lambda}\right)\right)}{\left(\frac{\delta}{\lambda}\right)^{k}} & \leq \frac{\mathscr{H}_{M}^{k}\left(B_{i} \cap B_{M}(f(x), \delta)\right)}{\delta^{k}} \\
& \leq \lambda^{2 k} \frac{\mathscr{H}_{N}^{k}\left(K_{i} \cap B_{N}(x, \delta \lambda)\right)}{(\delta \lambda)^{k}}
\end{aligned}
$$

Thus, taking advantage of the assumption that $x$ is a density point and letting $\delta$ go to zero and $\lambda$ go to one, we have the density is constant almost everywhere. The result follows.

We note that, if we had defined $\mathscr{H}_{N}^{k}$ with a normalizing constant, we would then have the analogue of the classical fact that an $N$-rectifiable set has density one almost everywhere.

Theorem 3.3. Suppose $f: N \rightarrow M$ is a Lipschitz map. Then, for any $\mathscr{H}_{N}^{k}$-measurable set $E$,

$$
\int_{E} J(n) d \mathscr{H}_{N}^{k}(n)=\int_{M} N\left(\left.f\right|_{E}, m\right) d \mathscr{H}_{M}^{k}(m)
$$

Proof: Assume for a moment that at every point in $E$, the Pansu differential is well defined and has trivial kernel. In other words, $E \subset\left\{x \mid \Delta_{x}\right.$ is a nondegenerate CC metric $\}$. Then, fixing $\lambda>1$ and using lemma 3.1. we find a countable cover $\left\{B_{i}\right\}$ with the approximative properties described in the lemma. Let $J_{i}=\frac{\mathscr{H}_{i}^{k}\left(B_{N}(x, 1)\right)}{\mathscr{H}_{N}^{k}\left(B_{N}(x, 1)\right)}$. Note that, under the assumption of nondegeneracy, $\mathscr{H}_{N}^{k}$ and $\mathscr{H}_{i}^{k}$ are both constant multiples of one another and $\mathscr{H}_{i}^{k}=$ $J_{i} \mathscr{H}_{N}^{k}$. Using these facts, we have that

$$
\lambda^{-k} J_{i} \leq J(x) \leq \lambda^{k} J_{i} \text { for all } x \in B_{i}
$$

and hence,

$$
\begin{aligned}
\lambda^{-2 k} \int_{B_{i} \cap E} J(n) d \mathscr{H}_{N}^{k}(n) & \leq \lambda^{-k} \int_{B_{i} \cap E} J_{i} d \mathscr{H}_{N}^{k}(n)=\lambda^{-k} \int_{B_{i} \cap E} d \mathscr{H}_{i}^{k} \\
& \leq \int_{M} N\left(\left.f\right|_{B_{i} \cap E}, m\right) d \mathscr{H}_{M}^{k}(m) \leq \lambda^{k} \int_{B_{i} \cap E} d \mathscr{H}_{i}^{k} \\
& =\lambda^{k} \int_{B_{i} \cap E} J_{i} d \mathscr{H}_{N}^{k}(n) \leq \lambda^{2 k} \int_{B_{i} \cap E} J(n) d \mathscr{H}_{N}^{k}(n)
\end{aligned}
$$

Thus, summing over $i$ and letting $\lambda \rightarrow 1$ we have the desired result.

By lemma 2.22 and the arguments used to prove it, we see that at points $x$ of $E$ where $\Delta_{x}$ is not defined or is degenerate, $J(x)=0$ and the set of all such $x$ maps to a $\mathscr{H}_{M}^{k}$ measure zero set, making both sides of the desired equation zero.

In light of the discussion above, we note that we could have defined the Jacobian via the Pansu differential as follows: at a point $x$ of Pansu differentiability, $J(x)=$ $\frac{\mathscr{H}_{M}^{k}\left(d f_{x}\left(B_{N}\left(e^{0}, 1\right)\right)\right)}{\mathscr{H}_{N}^{k}\left(B_{N}\left(e^{0}, 1\right)\right)}$. 


\section{CC-ReCtifiability}

Next, we introduce the definitions and basic properties of a theory of rectifiability for subsets of Carnot groups. One should view this analogously to the Euclidean case: Euclidean rectifiable sets are viewed as sets which have, in some sense, a manifold structure while CC-rectifiable sets will have a "manifold" structure where the local geometry modeled by general Carnot groups rather than simply by $\mathbb{R}^{n}$.

We begin with the relevant definitions. Assume that $N$ and $M$ are Carnot groups and that the Hausdorff dimension of $N$ is $k$.

Definition 4.1. Let $N$ be a Carnot group. A subset $E$ of another Carnot Group $\left(M, d_{M}\right)$ is said to be $N$-rectifiable if there exists $U$ an positive measure subset of $N$ and a Lipschitz map $f: U \rightarrow M$ such that $\mathscr{H}_{M}^{k}(E \backslash f(U))=0$. E is said to be countably $N$-rectifiable if there exist a countable number of $U_{i}$ and $f_{i}: U_{i} \rightarrow Y$ Lipschitz with $\mathscr{H}_{M}^{k}\left(E \backslash \cup_{i} f_{i}\left(U_{i}\right)\right)=0$.

As with the case of $\mathbb{R}^{n}$-rectifiability, we will be developing the notion of approximate tangent cones and their relation to rectifiability. Next, we wish to consider when a subset of $M$ is well approximated by $N$. If $i: N \rightarrow M$ is a graded group homomorphism, let $N(t)=\left\{x \mid d_{M}(x, i(N)) \leq t\right\}$.

Definition 4.2. A subset $E$ of $M$ is $N$-approximable at a point $a \in E$ if, for $\alpha>0$, there exist an injective homomorphism $i: N \rightarrow M$ with $i\left(e^{0}\right)=e^{0}$ and constants $r_{0}>0, \theta>0$ such that for any $0<r<r_{0}$,

$$
\mathscr{H}_{M}^{k}\left(E \cap B_{M}(b, \alpha r)\right) \geq \theta r^{k} \text { for } b \in a \cdot i(N) \cap B_{M}(a, r)
$$

and

$$
\mathscr{H}_{M}^{k}\left(E \cap B_{M}(a, r) \backslash a \cdot N(\alpha r)\right)<\alpha r^{k}
$$

$A$ set $E$ is called $N$-approximable if $\mathscr{H}_{M}^{k}$ a.e. point in $E$ is $N$-approximable.

Next, we have the first theorem, which follows from the differentiability of Lipschitz maps described in theorem 2.2. The proof of the theorem follows the argument of theorem 15.11 in Mat95 but with the appropriate changes for the Carnot case. The reader should also note that another key component of the proof relies on the fact that a Carnot group and its tangent cone at a point may be identified due to the existence of a homothety of the Carnot-Carathéodory metric thus allowing the homomorphism of tangent cones to be translated as a statement concerning sets in the group.

Theorem 4.3. Every countably $N$-rectifiable $E \subset M$ with nonzero $k$-dimensional Hausdorff measure is $N$-approximable.

Proof: In this proof, the main information to keep in mind is that the differentiability theory of Lipschitz maps between Carnot groups is close enough to that of Lipschitz maps between Euclidean spaces, allowing many of the Euclidean arguments to be used.

Fix $\varepsilon>0$. First, we reduce to an easier case. Let $0<\alpha<1$. Since $E$ is countably $N$-rectifiable, we consider an L-Lipschitz map between $E^{\prime} \subset N$ such that $f\left(E^{\prime}\right) \subset E$. To make the reduction to nicer sets, where the lower density is bounded away from zero, we use lemma 3.2 
Using this, we can cover $E^{\prime}$ up to a set of measure zero by a countable union of subsets, $S$, with the following properties. For each $S$, there exists constants $\theta>0, r_{0}>0$ such that for $x \in S$ and $0<r<r_{0}$,

$$
\mathscr{H}_{M}^{k}\left(f\left(E^{\prime}\right) \cap B_{M}(x, r)\right) \geq \theta r^{k}
$$

On these sets, we shall verify the properties of $N$-approximability.

To create a approximating isometric embedded copy of $N$, we use Pansu's differential mapping. Given a point $x$ at which $f$ is differentiable, Let $i_{x}(y)=f(x) d f_{x}\left(x^{-1} y\right)$ and $N_{x}=i_{x}(N)$. Note that the multiplication is multiplication in the Carnot group $M$. Since $d f_{x}$ is a group homomorphism, we know that $N_{x}$ is a subgroup of $M$ and from lemma 2.22 we know that for almost every such $x, N_{x}$ has large $k$-dimensional Hausdorff measure. In other words, the mapping $d f_{x}$ has, in some sense, full rank. Interpreting this result, we now show that at almost every image point of $f, N_{x}$ is a isomorphic copy of $N$. If it were not isomorphic, it would be isomorphic to a subgroup $N^{\prime}$ of $N$ (since $d f_{x}$ is a graded homomorphism). As a graded subgroup of $M, N_{x}$ inherits a Carnot-Carathéodory metric which is biLipschitz to the restricted Carnot-Carathéodory metric on $N^{\prime}$ because all Carnot-Carathéodory metrics given by varying the norm on the distribution of a single nilpotent Lie group are biLipschitz to one another. Thus, $N^{\prime}$ and $N_{x}$ have strictly lower Hausdorff dimension than $N$ and so lemma 2.22 shows this may only happen at almost every $f(x)$. Once again, we replace the set $E^{\prime}$ with a full measure subset of points $x$ such that $N_{x}$ has "full rank" in the sense described above. Thus, for each $x,\left(N_{x},\left.d_{M}\right|_{N_{x}}\right)$ is biLipschitz to $\left(N, d_{N}\right)$. Denote by $l(x)$ the lower Lipschitz constant for each $x$. By construction, $l(x)>0$ for all $x \in E^{\prime}$.

Recalling that the Lebesgue density theorem holds in $N$ for the measure $\mathscr{H}_{N}^{k}$ and using the approximations developed above, we will now find numbers $r_{0}>0$ and $\delta>0$ and a compact set $E_{0} \subset E^{\prime}$ with $\mathscr{H}_{N}^{k}\left(E^{\prime} \backslash E_{0}\right)<\varepsilon$ consisting only of density points of $E^{\prime}$ with nice approximative properties. We begin by picking a $\delta<\min \left\{\frac{\alpha}{4}, \frac{1}{L}\right\}$. First, pick a compact subset of the density points of $E^{\prime}, E_{0}$, so that for $x \in E_{0}, l(x) \geq 2 \delta$ (Property 1 ). Second, because $N_{x}$ approximates $f\left(E^{\prime}\right)$ well at $f(x)$ by lemma 2.19. I can pick $r_{0}$ small enough so that for $x \in E_{0}$,

$$
\left.d_{M}\left(f(y), i_{x}(y)\right)<\delta^{2} d_{N}(x, y) \text { for } y \in B_{N}\left(x, r_{0}\right) \quad \text { (Property } 2\right)
$$

So far, the choice of $r_{0}$ and $E_{0}$ depends on $\delta$ only. Last, we pick $\delta$ and, possibly readjusting $r_{0}$ to be smaller still, we may guarantee that for $x \in E_{0}, 0<r<r_{0}$ and $y \in B_{N}\left(x, \frac{r}{\delta}\right)$, $d_{N}\left(y, E_{0}\right)<\delta^{2} r$ (Property 3). Property 3 follows from the fact that all points in $E_{0}$ are density points of $E^{\prime}$.

Now, writing $E_{0}$ as the union of finitely many subsets $E_{i}$ with $\operatorname{diam}_{N}\left(C_{i}\right)<r_{0}$, we examine each $C_{i}$ individually. Consider a point $f(x)$ with $x \in C_{i}$ and $\Theta_{M}^{k}\left(f\left(E^{\prime}\right) \backslash f\left(C_{i}\right), f(x)\right)=$ 0 . Since almost every point in $f\left(C_{i}\right)$ has this property, we will consider only these points. Let $0<r<\frac{\delta r_{0}}{2}$ and pick $i_{x}(y) \in N_{x} \cap B_{M}(f(x), r)$. Note that $y \in B_{N}\left(x, \frac{r}{\delta}\right)$ by the property 1 . By property 3 above, there exists $z \in E^{\prime}$ such that $d_{N}(y, z)<\frac{2 r}{\delta}$. Using the triangle inequality, the fact that $i_{x}$ is $L$-Lipschitz (recall that $L<\frac{1}{\delta}$ ) and property 2, we get

$$
d_{M}\left(f(z), i_{x}(y)\right) \leq \delta^{2} d_{N}(x, z)+L d_{N}(y, z) \leq 3 r \delta
$$


Since $4 \delta<\theta$, we have from equation 3 that

$$
\mathscr{H}_{M}^{k}\left(f\left(E^{\prime}\right) \cap B_{M}\left(i_{x}(y), \alpha r\right)\right) \geq \mathscr{H}_{M}^{k}\left(f\left(E^{\prime}\right) \cap B_{M}(f(z), \delta r)\right) \geq \theta \delta^{k} r^{k}
$$

Taking $\lambda=\theta \delta^{k}$ we have verified equation 1 .

To verify equation 2, we observe the following:

First, by property 2 ,

$$
f\left(C_{i} \cap B_{N}\left(x, \frac{r}{\delta}\right)\right) \subset N_{x}(\delta r) \subset N_{x}(\alpha r)
$$

Second, using the lower Lipschitz bound on $C_{i}$ and property 2 again,

$$
f\left(C_{i} \backslash B_{N}\left(x, \frac{r}{\delta}\right)\right) \subset M \backslash B_{M}(f(x), r)
$$

and so, $f\left(C_{i}\right) \cap B_{M}(f(x), r)$ lies inside $N_{x}(\alpha r)$. Given the density assumption on $f(x)$, that $\Theta_{M}^{k}\left(f\left(E^{\prime}\right) \backslash f\left(C_{i}\right), f(x)\right)=0$, this implies that, again possibly shrinking $r_{0}$, that equation 2 holds.

\section{IMPLiCATIONS OF RECTIFIABILITY}

To do this, we need to use a type of projection mapping analogous to the projections onto planes used in the usual theory.

Definition 5.1. Let $V$ be a vector subspace of $\mathfrak{n}$ and let $\langle\cdot, \cdot\rangle$ be a Riemannian completion of the CC-inner product on $\mathfrak{n}$ which makes the grading orthogonal. Denote by $v^{\perp}$ the orthogonal complement of $V$ (with respect to this inner product). Let $\mathfrak{p r}_{V}: \mathfrak{n} \rightarrow V$ by the projection of $\mathfrak{n}$ onto $V$ and let $P_{V}: N \rightarrow e^{V}$ be the map exp $\circ \mathfrak{p r}_{V} \circ \exp { }^{-1}$ where exp is the usual exponential map. Also, let $Q_{V}$ be the map $P_{V^{\perp}}$. In each of the applications of the projection mappings below, there is an understood base point for the exponential map.

It is a direct consequence of the equivalence of the Carnot-Carathéodory metric and $d_{q n}$ that the projection defined above is a Lipschitz map only very rarely. Although the map may not be Lipschitz, the map defined above is a projection in the sense that $P_{V} \circ P_{V}$ is the identity map. One can easily construct examples of projections which are not Lipschitz. Note, however, that these projections are Hölder with exponent $\frac{1}{\operatorname{depth}(N)}$, where $\operatorname{depth}(N)$ is the numbers of levels in the grading of $\mathfrak{n}$, when we restrict our view to a neighborhood of the origin.

Example: Consider the 3-dimensional Heisenberg group, $H^{3}$, with a left invariant CarnotCarathéodory metric, $d_{c c}$. Let $V$ be the vector subspace spanned by the "Z" (nondistributional) direction. Then, the exponential image of $V$ in $H^{3}$ is a 1-parameter subgroup but $V$ is not a Lie subalgebra with compatible grading. We will now directly show that this projection is not Lipschitz. Not surprisingly, this stems from the nontrivial bracket structure. Giving $H^{3}$ coordinates $\{X, Y, Z\}$, we describe an element $e^{a X+b Y+c Z}$ by the triple $(a, b, c)$. Then $d_{c c}((a, b, c),(\alpha, \beta, \gamma))=d_{c c}\left((0,0,0),\left(\alpha-a, \beta-b, \gamma-c+\frac{1}{2}(\alpha b-a \beta)\right)\right)$. Fixing $\varepsilon>0$ and taking $\alpha=a+\varepsilon, \beta=b+\varepsilon$ and $a=\frac{2(\gamma-c)+b \varepsilon}{\varepsilon}$, this simplifies to 
$d_{c c}((0,0,0),(\varepsilon, \varepsilon, 0))=\varepsilon d_{c c}((0,0,0),(1,1,0))$. Computing the distance under the image of $P_{V}$, we have

$$
d_{c c}\left(P_{V}((a, b, c)), P_{V}((\alpha, \beta, \gamma))\right)=d_{c c}((0,0, c),(0,0, \gamma))=\sqrt{|\gamma-c|} d_{c c}((0,0,0),(0,0,1))
$$

Thus, since $\varepsilon, \gamma$ and $c$ are arbitrary, we see that the map cannot be Lipschitz. Notice, however, that if the two points are always within some fixed ball around the origin, this map is Hölder with exponent $\frac{1}{2}$

Definition 5.2. Let $V$ be a subspace of $\mathfrak{m}$. If $m_{0} \in M, 0<s<1$, and $0<R<\infty$, we define

$$
X\left(m_{0}, V, s\right)=\left\{m_{1} \in M \mid d_{M}\left(Q_{V}\left(m_{1}\right), Q_{V}\left(m_{0}\right)\right)<s d_{M}\left(m_{1}, m_{0}\right)\right\}
$$

Moreover, let

$$
X\left(m_{0}, R, V, s\right)=X\left(m_{0}, V, s\right) \cap B_{M}\left(m_{0}, R\right)
$$

The reader should look closely at this definitions of "cones". While they are the same in form as the Euclidean versions used by Mattila in Mat95, the actual objects look somewhat different in practice (it is useful and relatively easy to investigate the shape of these objects in the Heisenberg group). While they still have the same general form of an " $\mathrm{X}$ " emanating from the base point, the spreading of the limbs of the " $\mathrm{X}$ " is no longer quite linear. In practice, we will see that this will make no difference for our applications.

Next, we assume that $N$ and $M$ are Carnot groups of Hausdorff dimensions $k$ and $l$ respectively.

Definition 5.3. Suppose $Y \subset M, m \in M$ and $V$ is a subspace of $\mathfrak{m}$ with $\exp (V)$ isomorphic to $N$. We say that $\exp _{m}(V)$ is an approximate tangent cone for $Y$ at $m$ if $\Theta^{* k}(Y, m)>0$ and for all $0<s<1$,

$$
\lim _{r \rightarrow 0^{+}} \frac{\mathscr{H}_{M}^{k}\left(Y \cap B_{M}(m, r) \backslash X(m, V, s)\right)}{r^{k}}=0
$$

Following the Euclidean notation, we denote the set of all approximate tangent cones as $\operatorname{apTan}^{N}(Y, m)$. Note that this set depends on a choice of Carnot group $N$.

Next, we come to the first main theorem; we show that $N$-rectifiability implies $N$ approximability and the existence of approximate tangent cones almost everywhere.

Theorem 5.4. Let $N$ and $M$ be Carnot groups and the Hausdorff dimensions of $N$ is $k$. Let $Y$ be an $N$-rectifiable subset of $M$ with $\mathscr{H}_{M}^{k}(Y)<\infty$. Then,

(1) $Y$ is $N$-approximable.

(2) For $\mathscr{H}_{M}^{k}$ almost all $y \in Y$, there is a unique approximate tangent cone at $y$ isometric to $N$.

Proof: As with the previous results, this proof follows the development of [Mat95] with changes for the Carnot case.

(1) is theorem 4 , so it remains to prove the other implication.

$(1) \Rightarrow(2)$

Notice that, for $\varepsilon>0$ sufficiently small, $B_{M}(y, r) \backslash X(y, N, s) \subset\left(B_{M}(y, r) \backslash N(\varepsilon s r)\right) \cup$ $B_{M}(y, \varepsilon r)$. From this, and the assumption that $Y$ is $N$-approximable (use the second property in the definition), we see that a copy of $N$ is an approximate tangent cone for $Y$ 
at almost every point. To see that the approximate tangent cone is unique, we use property (1) in definition 4.2 and the definition of approximate tangent cone.

\section{Measures in CONES}

Theorem[5.4] stops short of proving the equivalence of $N$-rectifiability, $N$-approximability and the existence of approximate tangent cones. However, we will show that $N$-rectifiability is implied by the existence of a stronger notion of approximate tangent space. The notion of approximate tangent cone in the last section is not strong enough to easily characterize rectifiable sets due to the failure of the projections to be Lipschitz. In this section, we introduce a stronger version of approximate tangent cones.

Definition 6.1. Let $M$ be a Carnot group. Suppose $Y \subset M, m \in M$ and $V$ is a subspace of $\mathfrak{m}$ with $\exp (V)$ isomorphic to $N$, where $N$ is a subgroup of $M$. We say that $\exp _{m_{0}}(V)$ (for some $m_{0}$ in $M$ ) is a strong approximate tangent cone for $Y$ at $m$ if $\Theta^{* k}(Y, m)>0$ and for all $0<s<1$,

$$
\lim _{r \rightarrow 0^{+}} \frac{\mathscr{H}_{M}^{k}\left(Y \cap B_{M}(m, r) \backslash X(m, V, s)\right)}{r^{k d e p t h(N)}}=0
$$

and that for sufficiently small $s$ and $\varepsilon>0$,

$$
X(m, V, s) \cap X\left(m, V^{\perp}, s\right) \cap B_{M}(m, \varepsilon)=\emptyset
$$

We denote the set of all strong approximate tangent cones as $\operatorname{SapTan}^{N}(Y, m)$.

\section{Remarks:}

(1) This definition is highly restrictive and is certainly not the sharpest definition to have the consequence of $N$-rectifiability.

(2) Note that the group $N$ in the previous definition is now not restricted to being a Carnot group itself, it is merely a subgroup of a Carnot group.

We will need a method of producing Lipschitz maps. To do so, we follow the idea used in Mat95 of considering measures of the intersections of cones and the set $E$ to conclude $N$-rectifiability.

We begin with a simple lemma to aid in producing Lipschitz maps to rectifiable sets.

Lemma 6.2. Suppose $Y \subset M$ and $V$ is the orthogonal complement of a subspace $\mathfrak{n}$ of $\mathfrak{m}, 0<s<1$ and $0<R<\infty$ then if $Y \cap X(y, R, V, s)=\emptyset$ for all $y \in Y$, then $Y$ is $N$-rectifiable.

Proof: Geometrically, this says roughly that the Lie algebra preimage of the set $Y$ at each point locally lies in $\mathfrak{n}$. Thus it makes sense that one can approximate the set by Lipschitz images of pieces of $N$. To prove this, we first may assume that $\operatorname{diam}_{M}(Y)<R$, otherwise we may simply cut up $Y$ into a countable number of such pieces. We note that, for this lemma to be true, we must assume that the maps $Q_{V}$ are all the same mapping, i.e. that the projections are defined via the exponential map at the same basepoint (it does not matter now what basepoint we pick). Next, we observe that if $y_{1}, y_{2} \in Y$, the hypothesis of empty intersection implies that $d_{M}\left(Q_{V}\left(y_{1}\right), Q_{V}\left(y_{2}\right)\right)>s d_{M}\left(y_{1}, y_{2}\right)$. Thus, the map $\left(\left.Q_{V}\right|_{Y}\right)^{-1}$ is Lipschitz with constant less than $\frac{1}{s}$. We see that there exists $\bar{Y} \subset N$, a positive measure subset such that $\left(\left.Q_{V}\right|_{Y}\right)^{-1}: \bar{Y} \rightarrow Y$. Hence, $Y$ is $N$-rectifiable. 
The reader should note that one implication of the lemma is that a purely $N$-unrectifiable set must have a nonempty intersection with the cone for almost all points in the set.

Next, we refine the lemma above, replacing the requirement of empty intersection with a bound on the measure of the intersection.

Lemma 6.3. Let $V, \mathfrak{n}$ and $s$ be as above. Let $0<\delta<\infty$ and $0<\lambda<\infty$. Let $k$ be the Hausdorff dimension of $N$. If $Y \subset M$ is purely $N$-unrectifiable and $\mathscr{H}_{M}^{k}(Y \cap X(y, r, V, s)) \leq$ $\lambda(r s)^{k d e p t h(N)}$ for $y \in Y$ and $0<r<\delta$, then $\mathscr{H}_{M}^{k}\left(Y \cap B_{M}\left(m, \frac{\delta}{6}\right)\right) \leq C \lambda \delta^{k}$ for all $m \in M$ where $C$ is a constant depending only on $k$ and the structure of the Lie group $M$.

Proof: This proof is similar to the proof of lemma 15.14 in [Mat95. Through the usual reduction of $Y$ via decomposition into countably many sets, we may assume that $Y \subset$ $B_{M}\left(m, \frac{\delta}{6}\right)$. Recall also that $Q_{V}$ is a locally Hölder map with exponent at worst $\frac{1}{\text { depthM }}$. Let $c_{0}$ be the Hölder constant. By the previous lemma, we know that $Y \cap X\left(m, V, \frac{s}{4}\right) \neq \emptyset$ for almost every $m \in Y$. Throwing away a set of measure zero, we may assume every point in $Y$ enjoys this property. Next, Let $h(m)=\sup \left\{d_{M}(m, y) \mid y \in Y \cap X\left(m, V, \frac{s}{4}\right)\right\}$ when $m \in Y$. Pick $m_{1} \in Y \cap X\left(m, V, \frac{s}{4}\right)$ so that $d_{M}\left(m, m_{1}\right) \geq \frac{3 h(m)}{4}$. Further, we define

$$
C_{m}=Q_{V}^{-1}\left(Q_{V}\left(B_{M}\left(m, \frac{1}{c_{0}}\left(\frac{\operatorname{sh}(m)}{4}\right)^{\operatorname{depth}(M)}\right)\right)\right)
$$

We claim that $Y \cap C_{m} \subset X(m, 2 h(m), V, s) \cup X\left(m_{1}, 2 h(m), V, s\right)$ for each $m \in Y$. To prove this, suppose $y \in Y \cap C_{m}$. Since $Q_{V}$ is a Hölder map and our choice of $C_{m}$, we have

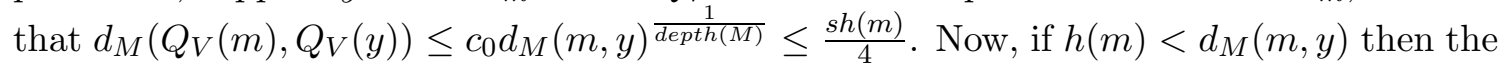
previous inequality and the fact the $y \in X\left(m, V, \frac{s}{4}\right)$ imply that $d_{M}(m, y) \leq h(m)$. Thus, $d_{M}(m, y) \leq h(m)$ and so $d_{M}\left(m_{1}, y\right) \leq 2 h(m)$. If, further, $y \notin X\left(m_{1}, 2 h(m), V, s\right)$ then,

$$
\begin{aligned}
s d_{M}\left(m_{1}, y\right) & \leq d_{M}\left(Q_{V}\left(m_{1}\right), Q_{V}(y)\right) \leq d_{M}\left(Q_{V}\left(m_{1}\right), Q_{V}(m)\right)+d_{M}\left(Q_{V}(m), Q_{V}(y)\right) \\
& <\frac{s}{4} d_{M}\left(m, m_{1}\right)+\frac{s h(m)}{4} \leq \frac{s h(m)}{2}
\end{aligned}
$$

Putting this together with the fact that $d_{M}\left(m, m_{1}\right)>\frac{s h(m)}{4}$, we have $d_{M}(m, y)>\frac{h(m)}{4} \geq$ $\frac{d_{M}\left(Q_{V}(m), Q_{V}(y)\right)}{s}$. In other words, $y \in X(m, 2 h(m), V, s)$.

From the hypothesis of the lemma, this gives

$$
\mathscr{H}_{M}^{k}\left(Y \cap C_{m}\right) \leq 2 \lambda(2 h(m) s)^{k \operatorname{depth}(M)}
$$

Using a standard Vitali covering argument, we can find a countable set $I \subset Y$ s.t. $\left\{Q_{V}\left(B_{M}\left(y, \frac{1}{c_{0}}\left(\frac{s h(y)}{20}\right)^{\operatorname{depth}(M)}\right)\right)\right\}_{y \in I}$ are disjoint and $Q_{V}(Y)$ is contained in the union of the projected balls with the same centers and five times the radius. Therefore $Y \subset \cup_{y \in I} C_{y}$ 
and

$$
\begin{aligned}
\mathscr{H}_{M}^{k}(Y) & \leq \sum_{y \in I} \mathscr{H}_{M}^{k}\left(Y \cap C_{y}\right) \leq 2 \lambda 2^{k \operatorname{depth}(M)} \sum_{y \in I}(h(y) s)^{k \operatorname{depth}(N)} \\
& =\frac{2^{k \operatorname{depth}(M)+1} \lambda 20^{k \operatorname{depth}(M)}}{\mathscr{H}_{M}^{k}\left(B_{M}\left(e^{0}, 1\right) \cap \exp (\mathfrak{n})\right)} \sum_{y \in I} \mathscr{H}_{M}^{k}\left(\exp (\mathfrak{n}) \cap B_{M}\left(Q_{V}(y),\left(\frac{\operatorname{sh}(y)}{20}\right)^{\operatorname{depth}(M)}\right)\right) \\
& \leq \frac{2^{k \operatorname{depth}(M)+1} \lambda 20^{k \operatorname{depth}(M)}}{\mathscr{H}_{M}^{k}\left(B_{M}\left(e^{0}, 1\right) \cap \exp (\mathfrak{n})\right)} \mathscr{H}_{M}^{k}\left(\exp (\mathfrak{n}) \cap B_{M}\left(Q_{V}(m), \frac{\delta}{2}\right)\right)=C(k, M) \lambda \delta^{k}
\end{aligned}
$$

Corollary 6.4. Let $V, \mathfrak{n}$ and $s$ be as in the last lemma. Suppose $Y \subset M$ is purely $N$ unrectifiable with $\mathscr{H}_{M}^{k}(Y)<\infty$, then there exists a constant $C$ depending only on $k$ and $\mathscr{H}_{N}^{k}\left(B_{N}\left(e^{0}, 1\right)\right)$ such that

$$
\mathscr{H}_{M}^{k}(Y \cap X(y, V, s), y) \geq C(s r)^{k \operatorname{depth}(M)}
$$

for $H_{M}^{k}$ a.e. $y \in Y$.

Proof: Again, this is the same as the proof of corollary 15.16 in Mat95 with the obvious modifications depending on the constant in lemma 6.3. The only detail which must be checked is the lower bound on the density of sets of positive measure at almost every point. This is covered in lemma 2.10.

Now, we can prove the following theorem.

Theorem 6.5. Suppose $Y$ is a $\mathscr{H}_{M}^{k}$-measureable subset of a Carnot group $M$ and $T$ is a subspace of $\mathfrak{m}$ so that $\exp (T)$ is isomorphic to $N$. Further suppose that for $\mathscr{H}_{M}^{k}$ a.e. $y \in Y$ $T$ is a strong approximate tangent cone at $y$. Then, $Y$ is $N$-rectifiable.

Proof: We will use lemma 6.3 to show this implication via contradiction. Thus, we assume $Y$ is purely $N$-unrectifiable and we will show that it can possess an approximate tangent cone isomorphic to $N$ satisfying the hypotheses almost nowhere. In this proof, we will identify $M$ with its lie algebra, denoted, for now, as $\mathbb{R}^{n}$. Let $k_{0}$ be the topological dimension of $N$ and let $G\left(n, k_{0}\right)$ be the space of $k_{0}$-planes in $\mathfrak{m}$ which are isomorphic to $\mathfrak{n}$. Let $m \in \mathbb{N}$ (we will adjust the choice of $m$ later in the proof). First, define the $\mathrm{CC}$ operator norm on $V, W \in G\left(n, k_{0}\right)$ by $\|V-W\|_{c c}=\sup _{\left\{u \mid d_{M}\left(e^{0}, e^{u}\right)<1\right\}} d_{M}\left(P_{V}\left(e^{u}\right), P_{W}\left(e^{u}\right)\right.$. Second, since the identity map $\left(M, d_{m}\right) \rightarrow \mathbb{R}^{n}$ is Lipschitz and the inverse is Hölder, we see that $G\left(n, k_{0}\right)$ is compact with respect to the CC operator norm as well as the standard operator norm. We can use the compactness of $G\left(n, k_{0}\right)$ with respect to the CC operator norm to cover $G\left(n, k_{0}\right)$ by finitely many balls of radius $\frac{1}{2 m}$. Call this family of balls $\mathcal{B}$. Consider now the subset $\mathcal{G}=\left\{B_{1}, \ldots, B_{l}\right\}$ of $\mathcal{B}$ of balls containing $k_{0}$-planes that, under the induced grading from $N$, are isomorphic to $N$. Let $G_{g r}\left(n, k_{0}\right) \subset G\left(n, k_{0}\right)$ be the subset of $k_{0}$-planes isomorphic to $N$ under the induced grading. Now, for each $B_{i}$, pick $W_{i} \in B_{i} \cap G_{g r}\left(n, k_{0}\right)$. Then, by construction, for $v \in B_{i} \cap G_{g r}\left(n, k_{0}\right)$, we know that $\left\|P_{V}-P_{W_{i}}\right\|_{c c}<\frac{1}{m}$. In other words, if $\alpha$ is any vector, we have

$$
d_{M}\left(P_{V}\left(e^{\alpha}\right), P_{W_{i}}\left(e^{\alpha}\right)\right) \leq C(m) d_{M}\left(e^{0}, e^{\alpha}\right)
$$


A NOTION OF RECTIFIABILITY MODELED ON CARNOT GROUPS

where $C(m)=$ const $\cdot\left(\frac{1}{m}\right)$. Here, we abuse notation and assume $V$ and $W_{i}$ are exponentiated at the same base point.

Fixing $W_{i}$, let $B=\left\{y \in Y \mid \exists V \in B_{i}\right.$ s.t. $V \in \operatorname{apTan}^{N}(Y, y)$ and $(+)$ holds $\}$. We want to show that $B$ has $\mathscr{H}_{M}^{k}$-measure zero. Suppose, on the contrary, that $B$ has positive measure. Since, by assumption, we know that every point in $B$ has a strong approximate tangent cone in $B_{i}$, given $\lambda>0, \exists r_{0}>0$ so that

$$
C=\left\{b \in B \mid \sup _{0<r<r_{0}} \frac{\mathscr{H}_{M}^{k}\left(B \cap B_{M}(b, r) \backslash X(b, V, C(m))\right)}{\left.r^{k \operatorname{depth}(N)}<\lambda\right\}}\right.
$$

has positive $\mathscr{H}_{M}^{k}$-measure.

Next, we claim that, for sufficiently large $m \in \mathbb{N}$,

$$
C \cap X\left(b, r, W_{i}^{\perp}, C(m)\right) \subset C \cap\left(B_{M}(b, r) \backslash X(b, V, C(m))\right)
$$

Geometrically, this is almost clear, but we shall prove it anyway. Suppose the claim does not hold; for every $m$, there exists $y \in C \cap\left(X\left(b, r, W_{i}^{\perp}, C(m)\right) \cap X(b, V, C(m))\right)$ and $y \neq b$. From the definitions of the "X" sets, we have

$$
\begin{gathered}
d_{M}\left(P_{W_{i}}(y), P_{W_{i}}(b)\right)<C(m) d_{M}(y, b) \\
d_{M}\left(P_{V^{\perp}}(y), P_{V^{\perp}}(b)\right)<C(m) d_{M}(y, b)
\end{gathered}
$$

From above, we know $d_{M}\left(P_{W_{i}}(y), P_{V}(y)\right) \leq C(m) d_{M}(b, y)$. Using the triangle inequality and (i) , we have

$$
d_{M}\left(P_{V}(y), P_{V}(b)\right)<3 C(m) d_{M}(y, b)
$$

Now, as $m \rightarrow \infty, C(m) \rightarrow 0$ so, considering (iii) and (iii), we can conclude that there exists an $0<s<1$ sufficiently small such that $y \in X(b, V, s) \cap X\left(b, V^{\perp}, s\right)$. By the second portion of the definition of strong approximate tangent cone, no such $y$ can exist. Picking $m$ so that the claim is true, we can now finish the proof of this implication using the following computation:

$$
\begin{aligned}
\mathscr{H}_{M}^{k}\left(C \cap X\left(b, r, W_{i}^{\perp}, C(m)\right)\right) & \leq \mathscr{H}_{M}^{k}\left(C \cap\left(B_{M}(b, r) \backslash X(b, V, C(m))\right)\right) \\
& \leq \lambda r^{k \operatorname{depth}(M)}
\end{aligned}
$$

Picking $\lambda$ sufficiently small, we violate corollary 6.4 Thus, $C$ has measure zero as does $B$ proving the theorem.

Remark: We reiterate that the class of $N$-rectifiable manifolds, for a Carnot group $N$, has good local properties. In particular, the tangent cone at each point (in this setting, it is easy to see using the $N$-approximability at a point that the unique approximate tangent cone will coincide with the tangent cone in the sense of Gromov) is isometric to $N$ in contrast to the examples cited earlier. This should allow for a stronger analysis of the local geometry of $N$-rectifiable smooth submanifolds. 


\section{Level Sets of $C_{N}^{1}$ Functions}

We turn now to a slightly different examination. Considering, for example, smooth submanifolds of Carnot groups, one is immediately led to a situation which is not covered in the previous discussion. Namely, the previous discussion considered only the case when the tangent objects were themselves Carnot groups. Considering submanifolds in the three dimensional Heisenberg group, one sees that the smooth tangent spaces (one possible local approximator) are not isomorphic to Carnot groups but to subgroups of Carnot groups (see the explicit example below).

To shed some light on this situation, this section investigates the properties of level sets of $C_{N}^{1}$ functions on a Carnot group, $N$. The main reason for focusing on this case is that it ties in nicely with the beautiful work of Franchi, Serapioni and Serra Cassano ([FSSC99]) who investigate rectifiability from a different point of view. In the Heisenberg group, $\mathbb{H}$, they show that a surface, $S$, of bounded perimeter can be decomposed as

$$
S=Z \bigcup \cup_{i=1}^{\infty} K_{i}
$$

where $Z$ is a set of perimeter zero and $K_{i}$ are compact subsets of noncharacteristic level sets of $C_{\mathbb{H}}^{1}$ functions. Thus, it is natural to ask whether level sets of $C_{N}^{1}$ functions possess rectifiability properties of the sort discussed in this paper near noncharacteristic points.

In modeling the local behavior of such sets, we will need to use subgroups of the Carnot group onto which the projection mapping is not Lipschitz. In fact, as in the Euclidean case, the subgroup which locally models $f^{-1}(t)$ at a point $x$ is $\operatorname{ker}\left(d f_{x}\right)$ which, by Pansu's differentiability theory, exists almost everywhere and is a subgroup for a.e. $x$.

We begin with an example illustrating that the process of constructing Lipschitz maps is quite delicate.

Example 7.1. Consider the mapping $f: H^{3} \rightarrow \mathbb{R}$ given by $e^{a X+b Y+c Z} \rightarrow\left(\left(|a|^{2}+|b|^{2}\right)^{2}+\right.$ $\left.|c|^{2}\right)^{\frac{1}{4}}$. This is a Lipschitz map (this is a quasi-norm on $H^{3}$, although it is slightly different than the quasi-norm defined in section 2, and hence is biLipschitz equivalent to the CarnotCarathéodory metric) and the inverse image of $t \in \mathbb{R}$ is the boundary of $B_{q n}\left(n_{0}, t\right)$. We will now calculate the kernels of the differential mappings at each point. First, we calculate $d f_{x}\left(e^{a X+b Y+c Z}\right)$. Fix $x=e^{\alpha X+\beta Y+\gamma Z}$. Since,

$$
x h_{r} e^{a X+b Y+c Z}=e^{(\alpha+r a) X+(\beta+r b) Y+\left(\gamma+r^{2} c+\frac{r}{2}(\alpha b-a \beta)\right) Z}
$$

we have

$$
\begin{gathered}
\lim _{r \rightarrow 0^{+}} \frac{\left(\left(|\alpha+r a|^{2}+|\beta+r b|^{2}\right)^{2}+\left|\gamma+r^{2} c+\frac{r}{2}(\alpha b-a \beta)\right|^{2}\right)^{\frac{1}{4}}-\left(\left(|\alpha|^{2}+|\beta|^{2}\right)^{2}+|\gamma|^{2}\right)^{\frac{1}{4}}}{r} \\
=\frac{\alpha^{3} a+\gamma \alpha b+\alpha \beta^{2} a-\gamma \beta a+\alpha^{2} \beta b+\beta^{3} b}{\left(\alpha^{4}+2 \alpha^{2} \beta^{2}+\beta^{4}+\gamma^{2}\right)^{\frac{3}{2}}}
\end{gathered}
$$

So, for this fixed $x$, we see that when $\gamma \alpha+\alpha^{2} \beta+\beta^{3} \neq 0$,

$$
\operatorname{ker}\left(d f_{x}\right)=\left\{e^{a X+b Y+c Z} \mid b=\frac{\gamma \beta-\alpha^{3}-\alpha \beta^{2}}{\gamma \alpha+\alpha^{2} \beta+\beta^{3}} a\right\}
$$


Note that these subgroups are all mutually isomorphic. One should note that the case when $\alpha=\beta=0$ is particularly interesting, for at those points, the kernel is $\left\{e^{a X+b Y+c Z}\right\}$. These are points where Pansu's differential is zero and hence are characteristic points of the level set. As mentioned above, we will be concerned only with neighborhoods of noncharacteristic points.

For this section, we make the following assumptions:

- $N$ is a Carnot group of (topological) dimension $n+m$, where $n$ is the dimension of the horizontal directions.

- $k$ denotes the Hausdorff dimension of $N$.

- $f: N \rightarrow \mathbb{R}$ is a $C_{N}^{1}$ function.

- For a fixed $t$, the characteristic set of $f^{-1}(t)$ is $\Sigma_{t}$.

The most natural candidate for a Lipschitz map between $U \subset N$ and $f^{-1}(t)$ would be, as in the Euclidean case, the inverse of the projection of $f^{-1}(t)$ to $\operatorname{ker}\left(d f_{x}\right)$. Unfortunately, this map is not always Lipschitz. We show this in a special case. Consider $x=e^{X}$ in $f^{-1}(1)$. Then, by the above calculation, $\operatorname{ker}\left(d f_{x}\right)=\left\{e^{b Y+c Z}\right\}$. Let $P_{x}: f^{-1}(1) \rightarrow \operatorname{ker}\left(d f_{x}\right)$ be the map $P_{x}\left(e^{a X+b Y+c Z}\right)=e^{b Y+c Z}$ where $\left(\left(|a|^{2}+|b|^{2}\right)^{2}+|c|^{2}\right)^{\frac{1}{4}}=1$. Taking $n_{1}=e^{a X+\sqrt{1-a^{4}} Z}$ and $n_{2}=e^{a X+\varepsilon Y+\sqrt{1-\left(a^{2}+\varepsilon^{2}\right)^{2}} Z}$ for $\varepsilon>0$ small and $a$ close to 1 , we have

$$
\begin{aligned}
d_{H^{3}}\left(n_{1}, n_{2}\right) & =d_{H^{3}}\left(e^{0}, e^{\varepsilon Y+\left(\sqrt{1-\left(a^{2}+\varepsilon^{2}\right)^{2}}-\sqrt{1-a^{4}}-\frac{1}{2} a \varepsilon\right) Z}\right) \\
& \geq C_{1} \varepsilon^{\frac{1}{2}}
\end{aligned}
$$

But, computing the distance between the projected points, we have

$$
\begin{aligned}
d_{H^{3}}\left(P_{x}\left(n_{1}\right), P_{x}\left(n_{2}\right)\right) & =d_{H^{3}}\left(e^{0}, e^{\varepsilon Y-\left(\sqrt{1-\left(a^{2}+\varepsilon^{2}\right)^{2}}-\sqrt{1-a^{4}}\right) Z}\right) \\
& \leq C_{2} \varepsilon
\end{aligned}
$$

Taking the quotient and applying the above estimates yields that $\operatorname{Lip}\left(P_{x}^{-1}\right) \geq C_{3} \varepsilon^{-\frac{1}{2}}$. Letting $\varepsilon$ go to zero shows that the inverse map cannot be Lipschitz.

In the theory of $\mathbb{R}^{n}$-rectifiability, one has a nice decomposition of Lipschitz maps between Euclidean spaces controlled by the area and co-area formulae. In particular, one can conclude that the inverse image of a point under a Lipschitz map is $\mathbb{R}^{k}$-rectifiable. We prove, in this section, that level sets of $C_{N}^{1}$ maps from $N$ to $\mathbb{R}$ are $T_{x}$-approximable at $x$, a nonchracteristic point, and have approximate tangent cones isomorphic to $T_{x}$ at $x$ for $T_{x}$ some subgroup of $N$. The proof of such a fact is significantly harder than in the Euclidean case because many of the tools (even the ones used in the previous sections) are not available in the cases where the local model for the level sets are subgroups of Carnot groups with incompatible gradings. To circumvent the standard arguments, we employ an approximation of the $C_{N}^{1}$ function by a smooth function using the usual mollification procedure. This gives us two tools - the smooth approximation and the Pansu differential of the function. Combining these two different perspectives allows us the desired control.

Lemma 7.2. If $f: N \rightarrow \mathbb{R}$ is a $C_{N}^{1}$ map, there exist smooth functions $f_{i}: N \rightarrow \mathbb{R}$ which converge uniformly to $f$ on compact sets. In addition, if $X$ is a horizontal vector field on $N$, then $X f_{i} \rightarrow X f$ uniformly on compact sets as $X f$ is continuous. 
Remarks on proof: This is a simple consequence of the convergence properties of convolution operators on graded nilpotent Lie groups and the usual mollification and approximation procedures. As in the Euclidean case, one has an approximation continuous functions by smooth functions which converge uniformly on compact sets. These facts are well known and can be found in, for example, [FS82. The reader should also see GN96, FSSC95. and FSSC99 (in particular proposition 5.8 and theorem 6.4) for an explicit discussion of mollifiers acting on functions on Carnot-Carathéodory spaces.

Lemma 7.3. For almost every $t \in \mathbb{R}, f_{i}^{-1}(t)$ are all smooth submanifolds of $N$.

Proof: This is an application of Sard's theorem.

Lemma 7.4. For $\mathscr{H}_{N}^{k}$ a.e. $t \in \mathbb{R}$, almost every point of $f_{i}^{-1}(t)$ is a point of Pansu differentiability for $f$. For almost every $t \in \mathbb{R}, f^{-1}(t)$ is $\mathscr{H}_{N}^{k-1}$ measurable and, for such a $t$, almost every $x \in f^{-1}(t)$ is a point of Pansu differentiability for $f$.

Proof: The first statement is a consequence of the coarea formula for smooth real valued maps on Carnot groups. See proposition 2.13 and Hei95] section 4.7. The second statement follows directly from lemma 2.10.15 in [Fed69].

Theorem 7.5. Let $N$ be a Carnot group of Hausdorff dimension $k$ and let $f: N \rightarrow \mathbb{R}$ be a $C_{N}^{1}$ map. Then, for a.e. $t \in \mathbb{R}$ and $\mathscr{H}_{N}^{k-1}$ a.e. $x \in \operatorname{Int}\left(f^{-1}(t) \backslash \Sigma_{t}\right)$, there exists a subgroup $T_{x} \subset N$ and $r_{x}>0$ such that $B_{N}\left(x, r_{x}\right) \cap\left(f^{-1}(t) \backslash \Sigma_{t}\right)$ is $T_{x}$-approximable at $x$ and $f^{-1}(t)$ has a unique approximate tangent cone isomorphic to $T_{x}$ at $x$.

The proof of this theorem follows from the next lemmas and lemma 7.11 .

Lemma 7.6. Suppose $x \in f^{-1}(t)$ is a point of Pansu differentiability for $f$. Let $T_{x}=$ $x \cdot \operatorname{ker}\left(d f_{x}\right)$. If $x^{\prime} \in f^{-1}(t) \cap B_{N}(x, s)$ then $d_{N}\left(x^{\prime}, T_{x}\right)=o(s)$.

Proof: Consider a one parameter family of points $x h_{s} e^{w_{1}(s)+w_{2}(s)} \in f^{-1}(t)$ where $w_{1}(s) \in$ $\operatorname{ker}\left(d f_{x}\right), w_{2}(s)$ is perpendicular to the kernel and $d_{N}\left(e^{0}, e^{w_{1}(s)+w_{2}(s)}\right)=1$ for all $s$. Now, by the choice of these points $\left|f\left(x h_{s} e^{w_{1}(s)+w_{2}(s)}\right)-f(x)\right|=0$. Moreover, using the Pansu differentiability at the point $x,\left|f\left(x h_{s} e^{w_{1}(s)+w_{2}(s)}\right)-\left(f(x)+s d f_{x}\left(e^{w_{2}(s)}\right)\right)\right|=o(s)$ and so, using the left invariance and homothety in the target, we have:

$$
\left|d f_{x}\left(e^{w_{2}(s)}\right)\right|=o(1)
$$

Moreover, we notice that $\left.d f_{x}\right|_{N / k e r\left(d f_{x}\right)}$ is biLipschitz since $\left.d_{N}\right|_{N / k e r\left(d f_{x}\right)}$ and $\left.d_{\mathbb{R}}\right|_{\operatorname{Im}\left(d f_{x}\right)}$ are simply nondegenerate metrics on $\mathbb{R}$ and $d f_{x}$ is a Lipschitz group homomorphism respecting dilations. Using this, we have that

$$
d_{N}\left(e^{w_{2}(s)}, e^{0}\right)=o(1)
$$

So, using the Campbell-Baker-Hausdorff formula and the fact that $w_{2}(s)$ lies in a Lie subalgebra, $d_{N}\left(x h_{s} e^{w_{1}(s)+w_{2}(s)}, x \cdot \operatorname{ker}\left(d f_{x}\right)\right)=d_{N}\left(h_{s} e^{w_{2}(s)}, e^{0}\right)=o(s)$.

Note that this lemma is vacuous at a characteristic point, where $\operatorname{ker}\left(d f_{x}\right)=N$.

Recalling the definition of Ahlfors regularity (definition 2.7) and the fact that smooth hypersurfaces of $N$ have Hausdorff dimension $k-1$ (lemma 2.6), we show the Ahlfors regularity of smooth level sets. We remark that a much more general version of this lemma is proved by R. Monti and D. Morbidelli in [MM02. We include the proof here for completeness. 
Lemma 7.7. Suppose $S$ is the level set of a smooth realvalued function on $N$ and let $\Sigma$ be its characteristic locus. If $x \in S \backslash \Sigma$ then there exists $r>0$ so that the metric space $\left(\overline{B_{N}(x, r) \cap S}, d_{N}\right)$ is Ahlfors regular of dimension $k-1$ with constants depending only on $x$. In other words, there exists a constant $A$ so that for $y \in \overline{B_{N}(x, r) \cap S}$ and $r>s>0$,

$$
A^{-1} s^{k-1} \leq \mathscr{H}_{N}^{k-1}\left(B_{N}(y, s) \cap\left(\overline{B_{N}(x, r) \cap S}\right)\right) \leq A s^{k-1}
$$

Proof: To set up notation, we assume $S$ is a smooth hypersurface given as a level set of a smooth function $f: N \rightarrow \mathbb{R}$, i.e. $S=f^{-1}(0)$. Let $\left\{X_{1}, \ldots, X_{n}\right\}$ be an orthonormal basis for the horizontal subbundle of $N$. Let $\left\{x_{i}\right\}$ be coordinates associated to $\left\{X_{i}\right\}$ and let $\left\{z_{1}, \ldots, z_{m}\right\}$ be coordinates in the nonhorizontal directions. Since $x \in S \backslash \Sigma$, $\nabla_{0} f$ does not vanish at $x$. Then, we can find a horizontal vector field, $V$, such that $V f(x) \neq 0$. Without loss of generality, we may take $V=X_{1}$. Using the (standard) implicit function theorem, we can find a smooth function $g\left(x_{2}, \ldots, x_{n}, z_{1}, \ldots, z_{m}\right)$ such that $f\left(g\left(x_{2}, \ldots, z_{m}\right), x_{2}, \ldots, z_{m}\right)=0$, for points in some neighborhood of $x$. In other words, $S$ is parameterized via $g$ :

$$
\begin{aligned}
\bar{g}: U \subseteq \mathbb{R}^{n+m-1} \longrightarrow S \subseteq N \cong \mathbb{R}^{n+m} \\
\quad\left(x_{2}, \ldots, x_{n}, z_{1}, \ldots, z_{m}\right) \mapsto\left(g\left(x_{2}, \ldots, z_{m}\right), x_{2}, \ldots, z_{m}\right) .
\end{aligned}
$$

With this notation in place, we now estimate the $\mathscr{H}_{N}^{k-1}$ measure of sets of the form $\operatorname{Box}(y, r) \cap S$.

First, pick a closed box around $x, C_{x}=\overline{B o x(x, r) \cap S}$ inside $\overline{g(U)}$ so that $\nabla_{0} f$ and $f_{x_{1}}$ do not vanish on $C_{x}$. Then, $\exists$ positive constants $K_{1}, K_{2}$ s.t.

$$
K_{1} \leq\left|\frac{\nabla_{0} f}{f_{x_{1}}}\right| \leq K_{2} \quad \text { for } y \in C_{x} .
$$

Now, for $y \in C_{x}$, consider $\operatorname{Box}(y, s) \cap S \subseteq C_{x}$. By proposition 2.14

$$
\mathscr{H}_{N}^{k-1}\left(\operatorname{Box}(y, s) \cap S \cap C_{x}\right)=\int_{B o x(y, r) \cap S \cap C_{x}}\left|\frac{\nabla_{0} f}{\nabla f}\right| d A
$$

where $d A$ is the Riemannian area element.

To compute $d A$, we need to compute the determinant of the metric matrix. First, note that $\bar{g}_{x_{i}}=\left(g_{x_{i}}, 0, \ldots, 1, \ldots, 0\right)$. Taking the matrix $G=\left(<\bar{g}_{x_{i}}, \bar{g}_{x_{j}}>\right)$ where $<\cdot, \cdot>$ is the Riemannian inner product on $N$. Note that, in the coordinates we are using for $N$, $<\cdot, \cdot\rangle$ is the standard Euclidean inner product. Thus,

$$
G=\left(\begin{array}{cccc}
g_{x_{2}}^{2}+1 & g_{x_{2}} g_{x_{3}} & \ldots & g_{x_{2}} g_{z_{m}} \\
g_{x_{3}} g_{x_{2}} & g_{x_{3}}^{2}+1 & \ldots & g_{x_{3}} g_{z_{m}} \\
& \ldots & \ldots & \\
g_{z_{m}} g_{x_{2}} & g_{z_{m}} g_{x_{3}} & \ldots & g_{z_{m}}^{2}+1
\end{array}\right)
$$

Computing $d A$ yields;

$$
\left(g_{x_{2}}^{2}+\cdots+g_{z_{m}}^{2}+1\right)^{\frac{1}{2}} d x_{1} \ldots d x_{2 n} d z_{1} \ldots d z_{m} .
$$

Using the implicit function theorem, we calculate that $g_{x_{j}}=-\frac{f_{x_{j}}}{f_{x_{1}}}$ for $n \geq j>1$ and $g_{z_{i}}=-\frac{f_{z_{i}}}{f_{x_{1}}}$ for $1 \leq i \leq m$. So, $d A=\frac{1}{\left|f_{x_{1}}\right|} \cdot|\nabla f| d x_{2} \ldots d z_{m}$. Thus, $K_{1} d x_{2} \ldots d z_{m} \leq$ 
$\left|\frac{\nabla_{0} f}{\nabla f}\right| d A \leq K_{2} d x_{2} \ldots d z_{m}$. To conclude the computation, we need to find a description of $\operatorname{Box}(y, s) \cap S \cap C_{x}$. Since we know bounds on $|\nabla f|$, it follows that there exists a constant $K_{3}$ (in fact, we may take $K_{3}$ to be one) so that if

$$
R_{1}=\left[-\frac{1}{K_{3}} s, \frac{1}{K_{3}} s\right]^{n-1} \times \ldots \times\left[-\left(\frac{1}{K_{3}} s\right)^{d(m)},\left(\frac{1}{K_{3}} s\right)^{d(m)}\right]
$$

then,

$$
\operatorname{Box}(y, s) \cap S \cap C_{x} \subset\left\{\left(g\left(x_{2}, \ldots, z_{m}\right), x_{2}, \ldots, z_{m}\right) \mid\left(x_{2}, \ldots, z_{m}\right) \in R_{1}\right\}
$$

Applying equation (*), we have

$$
\mathscr{H}_{N}^{k-1}\left(\operatorname{Box}(y, s) \cap S \cap C_{x}\right) \leq K_{2} \int_{R_{1}} d x_{2} \ldots d z_{m}=2 K_{2} \frac{s^{k-1}}{K_{3}^{k-1}}
$$

To estimate $\mathscr{H}_{N}^{k-1}\left(\operatorname{Box}(y, s) \cap S \cap C_{x}\right)$ from below, we notice that, in the worst case, $y$ lies on the boundary of $\operatorname{Box}(x, r)$. Thus, there is at least a quarter of $B o x(y, s)$ inside of $\operatorname{Box}(x, r)$. Using the same reasoning as above, there exists a constant $K_{4}$ so that if

$$
R_{2}=\left[-\frac{1}{K_{4}} s, \frac{1}{K_{4}} s\right]^{n-1} \times \ldots \times\left[-\left(\frac{1}{K_{4}} s\right)^{d(m)},\left(\frac{1}{K_{4}} s\right)^{d(m)}\right]
$$

then,

$$
\left\{\left(g\left(x_{2}, \ldots, z_{m}\right), x_{2}, \ldots, z_{m}\right) \mid\left(x_{2}, \ldots, z_{m}\right) \in R_{2} \subset \operatorname{Box}(y, s) \cap S\right\}
$$

and a quarter of this set lies inside $\operatorname{Box}(y, s) \cap S \cap C_{x}$.

Again, using equation $(*)$, we have

$$
\frac{K_{1}}{2 K_{4}^{k-1}} s^{k-1} \leq \mathscr{H}_{N}^{k-1}\left(\operatorname{Box}(y, s) \cap S \cap C_{x}\right)
$$

To summarize, this says that the metric space $\left(C_{x}, d_{q n}\right)$ is an Ahlfors regular space of dimension $k-1$ where the constant is $\max \left\{\frac{2 K_{2}}{K_{3}^{k-1}}, \frac{2 K_{4}^{k-1}}{K_{1}}\right\}$.

Now by the Ball-Box theorem, we have that

$$
\begin{array}{r}
\frac{K_{1}}{2\left(C K_{4}\right)^{k-1}} s^{k-1} \leq \mathscr{H}_{N}^{k-1}\left(\operatorname{Box}_{N}\left(y, C^{-1} s\right) \cap S \cap C_{x}\right) \leq \mathscr{H}_{N}^{k-1}\left(B_{N}(y, s) \cap S \cap C_{x}\right) \\
\leq \mathscr{H}_{N}^{k-1}\left(\operatorname{Box}_{N}(y, C s) \cap S \cap C_{x}\right) \leq \frac{2 K_{2} C^{k-1}}{K_{3} C^{k-1}} s^{k-1}
\end{array}
$$

In other words $\left(C_{x}, d_{N}\right)$ is an Ahlfors regular space of dimension $k-1$. Again using the Ball-Box theorem and potentially adjusting $r$, we see that $\left(\overline{B_{N}(x, r) \cap S}, d_{N}\right)$ is Ahlfors regular of dimension $k-1$ with constant $A$ depending on $\left\{C, K_{1}, K_{2}, K_{3}, K_{4}\right\}$. Since, $C$ is a universal constant (for $N$ ) and the $K_{i}$ depend only on the point $x$, we have the desired result.

Remark: Suppose $x$ is a noncharacteristic point in $f^{-1}(t)$ with the property that there exists a neighorhood of $x$ with no characteristic points. Consider the case when we have 
smooth functions $f_{i}$ approximating a $C_{N}^{1}$ function $f$. Then, using lemma [7.6] for sufficiently large $i$ (i.e. when the approximation is close enough), we can pick the constants $K_{3}$ and $K_{4}$ uniformly in $i$. Also, since $\nabla_{0} f_{i}$ converges to $\nabla_{0} f$, we can pick the constants $K_{1}$ and $K_{2}$ uniformly as well.

Lemma 7.8. Fix a compact set $\Omega \subset N$. Let $C=\left(f^{-1}(t) \backslash \Sigma_{t}\right) \cap \Omega$ and $C_{i}=f_{i}^{-1}(t) \cap \Omega$. Then, there exists a constant $\kappa>0$ such that for $x \in N$ and $r>0$,

$$
\kappa \mathscr{H}_{N}^{k-1}\left(C \cap B_{N}(x, r)\right) \geq \varlimsup_{i \rightarrow \infty} \mathscr{H}_{N}^{k-1}\left(C_{i} \cap B_{N}(x, r)\right)
$$

Proof: This is not a hard fact to prove, but we cite it as a special case of lemma 8.35 in DS97. First we note that by the previous lemma, $C_{i} \cap B_{N}(x, r)$ is Ahlfors regular of dimension $k-1$. Second, by the remark after the previous lemma, the constants can be picked universally for large enough $i$. Thus, the hypotheses of lemma 8.35 in [DS97] are satisfied.

In the next lemma, we use the notation $\operatorname{Int}(C)$ to denote the interior of a set $C$.

Lemma 7.9. Fix $\alpha>0$. Then, for $x \in \operatorname{Int}\left(f^{-1}(t) \backslash \Sigma_{t}\right)$, there exists $s_{0}>0, \theta>0$ such that for $0<s<s_{0}$ and $x^{\prime} \in T_{x} \cap B_{N}(x, s)$ then, $\mathscr{H}_{N}^{k-1}\left(f^{-1}(t) \cap B_{N}\left(x^{\prime}, \alpha s\right)\right) \geq \theta s^{k-1}$.

Proof: To determine $s_{0}$, we use lemma [7.6] The estimate in this lemma and the triangle inequality tell us that if $x^{\prime} \in T_{x} \cap B_{N}(x, s)$ then $d_{N}\left(x^{\prime}, f^{-1}(t)\right)=o(s)$. Picking $s_{0}$ small enough (this will depend on $\alpha$ ), we can guarantee that $B_{N}\left(x^{\prime}, \frac{\alpha s}{2}\right) \cap f^{-1}(t) \neq \emptyset$ for $0<s<s_{0}$ and $x^{\prime} \in T_{x} \cap B_{N}(x, s)$. Let $B_{s}^{i}=B_{N}\left(x^{\prime}, \alpha s\right) \cap f_{i}^{-1}(t)$. We know from the construction of the $f_{i}$ that the $B_{s}^{i}$ converge to $B_{s}=B_{N}\left(x^{\prime}, \alpha s\right) \cap f^{-1}(t)$. Therefore, by lemma [7.8, $\varlimsup_{i \rightarrow \infty} \mathscr{H}_{N}^{k-1}\left(B_{s}^{i}\right) \leq \kappa \mathscr{H}_{N}^{k-1}\left(B_{s}\right)$. Also, in lemma 7.7] we noted that $f_{i}^{-1}(t) \cap \Omega$ is Ahlfors regular. By our choice of $s_{0}$, we know that, for sufficiently all large $i$, there are points $x_{i} \in f_{i}^{-1}(t) \cap \Omega$ such that $B_{N}\left(x_{i}, \frac{\alpha s}{4}\right) \cap f_{i}^{-1}(t) \subset B_{s}^{i}$. By Ahlfors regularity, we have $\mathscr{H}_{N}^{k-1}\left(B_{s}^{i}\right) \geq \frac{K \alpha^{k-1} s^{-k-1}}{4^{k-1}}$. Moreover, the remark after lemma 7.7 allows us to pick a constant $K$ independent of $i$, yielding the desired estimate.

Proof of theorem 7.5: As in lemma 7.6, we let $T_{x}=x \cdot \operatorname{ker}\left(d f_{x}\right)$. Next, for this candidate local structure, we must verify conditions (1) and (2) in definition 4.2. Condition (1) is verified by lemma [7.9, Note that the constant $r_{x}$ in the statement of theorem is given by the constant $s_{0}$ in lemma 7.9, Moreover, a consequence of lemma 7.6, there exists $r_{0}>0$ such that $\left(f^{-1}(t) \cap B_{N}(x, r)\right) \backslash T_{x}(\alpha r)=\emptyset$ for $0<r<r_{0}$. The proof the existence of a unique approximate tangent cone isometric to $N$ follows just as in one implication of theorem 5.4 .

Remark: The techniques and computations in this section are very similar in spirit to those used to prove the implicit function theorem (on the Heisenberg group) in [FSSC99. The author is grateful to B. Franchi, R. Serapioni, and F. Serra Cassano for providing an early version of [FSSC99] as well as valuable conversations concerning their techniques.

Next, we note that if the map $f$ is suitably generic at $x$, then a neighborhood of $x$ is $T_{x}$-approximable. Let $\mathfrak{n}$ be the Lie algebra of $N$ and suppose $\left\{X_{1}, \ldots, X_{n}\right\}$ is a basis for 
$\mathcal{V}$. We define $O \subset N$ by

$$
O=\left\{a_{1} X_{1}+\cdots+a_{n} X_{n} \mid a_{i} \neq 0 \text { for all } i\right\}
$$

Definition 7.10. Fix $t \in \mathbb{R}$. A $C_{N}^{1}$ map $f: N \rightarrow \mathbb{R}$ is generic at $x \in f^{-1}(t)$ if $\nabla_{0} f(x) \in O$.

Lemma 7.11. Suppose $\Omega_{t}$ is a compact subset of $f^{-1}(t) \backslash \Sigma_{t}$ and suppose $f$ is generic at $x \in \Omega_{t}$. Then, there exists $r_{x}>0$ so that for each $y \in B_{N}\left(x, r_{x}\right) \cap \Omega_{t}, k e r\left(d f_{y}\right)$ and $\operatorname{ker}\left(d f_{x}\right)$ are isomorphic as subgroups of $N$.

Proof: The exponential preimage of $\operatorname{ker}\left(d f_{x}\right)$ in the Lie algebra based at $x$ is a vector subspace of $\mathfrak{n}$. Denote this subspace by $V_{x}$. Recall that the Lie algebra $\mathfrak{n}$ is graded, i.e.

$$
\mathfrak{n}=n_{1} \oplus n_{2} \oplus \cdots \oplus n_{l}
$$

We define the induced grading data on $V_{x}$ by the decomposition

$$
V_{x}=V_{x} \cap n_{1} \oplus V_{x} \cap n_{2} \oplus \cdots \oplus V_{x} \cap n_{l}
$$

We denote $V_{x} \cap n_{i}$ by $V_{x}^{i}$. We say that two subspaces, $V_{x}$ and $V_{y}$ have compatible induced grading data if there is a algebraic isomorphism between $V_{x}$ and $V_{y}$ mapping $V_{x}^{i}$ to $V_{y}^{i}$ for all $i$.

Now, the subgroups $\operatorname{ker}\left(d f_{x}\right)$ and $\operatorname{ker}\left(d f_{y}\right)$ are isomorphic and biLipschitz with respect to the quasi-norm of $N$ restricted to the respective subgroups if $V_{x}$ and $V_{y}$ have compatible induced grading data. In this case, we say that $\operatorname{ker}\left(d f_{x}\right)$ is equivalent to $\operatorname{ker}\left(d f_{y}\right)$. By the assumption of genericity and the continuity of $d f_{x}=\nabla_{0} f(x)$, we see that there exists an $r_{x}>0$ so that $\nabla_{0} f(y) \in O$ for all $y \in B_{N}\left(x, r_{x}\right) \cap f^{-1}(t)$. Now, fixing a $y \in B_{N}\left(x, r_{x}\right) \cap$ $f^{-1}(t)$ we can find constants so that

$$
\begin{aligned}
& V_{x}=\operatorname{span}\left\{a_{2} X_{1}-X_{2}, \ldots, a_{n} X_{1}-X_{n}\right\} \\
& V_{y}=\operatorname{span}\left\{b_{2} X_{1}-X_{2}, \ldots, b_{n} X_{1}-X_{n}\right\}
\end{aligned}
$$

for nonzero constants $\left\{a_{i}, b_{i}\right\}$. We realize the compatibility between $V_{x}$ and $V_{y}$ via the map which takes $a_{i} X_{1}-X_{i}$ to $b_{i} X_{1}-X_{i}$. Thus $k e r\left(d f_{x}\right)$ and $k e r\left(d f_{y}\right)$ are equivalent subgroups of $N$.

Corollary 7.12. Using the notation above, if $f$ is generic at $x \in f^{-1}(t)$ then there exists a neighborhood, $E$, of $x$ in $f^{-1}(t)$ and a subgroup $T_{x}$ of $N$ so that $E$ is $T_{x}$-approximable.

Proof: This follows directly from the previous lemma and the previous theorem.

\section{REFERENCES}

[AK99a] Luigi Ambrosio and Bernd Kirchheim. Currents in metric spaces Acta Math., 185(1):1-80, 2000.

[AK99b] Luigi Ambrosio and Bernd Kirchheim. Rectifiable sets in metric and Banach spaces. Math. Ann.,318 (3):527-555, 2000.

[Amb] Luigi Ambrosio. Fine properties of sets of finite perimeter in doubling metric measure spaces. Set-Valued Anal., 10(2-3):111-128, 2002.

[Bal02] Zoltan Balogh Size of characteristic sets and functions with prescribed gradients. To appear in J. für die Reine und Angewandte Mathematik.

[Bel96] André Bellaïche. The tangent space in sub-Riemannian geometry. In Sub-Riemannian geometry, pages 1-78. Birkhäuser, Basel, 1996. 
[Che99] J. Cheeger. Differentiability of Lipschitz functions on metric measure spaces. Geom. Funct. Anal., 9(3):428-517, 1999.

[D'A95] Giuseppina D'Ambra. Nash $C^{1}$-embedding theorem for Carnot-Carathéodory metrics. Differential Geom. Appl., 5(2):105-119, 1995.

[DS97] Guy David and Stephen Semmes. Fractured fractals and broken dreams. The Clarendon Press, Oxford University Press, New York, 1997. Self-similar geometry through metric and measure.

[Fed69] Herbert Federer. Geometric measure theory. Springer-Verlag New York Inc., New York, 1969. Die Grundlehren der mathematischen Wissenschaften, Band 153.

[FS82] G. B. Folland and Elias M. Stein. Hardy spaces on homogeneous groups. Princeton University Press, Princeton, N.J., 1982.

[FSSC95] Bruno Franchi, Raul Serapioni, and Francesco Serra Cassano. Champs de vecteurs, théorème d'approximation de Meyers-Serrin et phénomène de Lavrentev pour des fonctionnelles dégénérées. C. R. Acad. Sci. Paris Sér. I Math., 320(6):695-698, 1995.

[FSSC99] Bruno Franchi, Raul Serapioni, and Francesco Serra Cassano. Rectifiability and Perimeter in the Heisenberg group. Math. Ann., 321(3):479-531,2001.

[GN96] Nicola Garofalo and Duy-Minh Nhieu. Isoperimetric and Sobolev inequalities for CarnotCarathéodory spaces and the existence of minimal surfaces. Comm. Pure Appl. Math., 49(10):1081-1144, 1996.

[Goo76] Roe W. Goodman. Nilpotent Lie groups: structure and applications to analysis. Springer-Verlag, Berlin, 1976. Lecture Notes in Mathematics, Vol. 562.

[Gro96] Mikhael Gromov. Carnot-Carathéodory spaces seen from within. In Sub-Riemannian geometry, volume 144 of Progr. Math., pages 79-323. Birkhäuser, Basel, 1996.

[Hei95] Juha Heinonen. Calculus on Carnot groups. In Fall School in Analysis (Jyväskylä, 1994), pages 1-31. Univ. Jyväskylä, Jyväskylä, 1995.

[Kir94] Bernd Kirchheim. Rectifiable metric spaces: Local structure and the regularity of the Hausdorff measure. Proc. Amer. Math. Soc., 121:113-123, 1994.

[Mag01] Valentino Magnani. Differentiability and area formula on stratified Lie groups. Houston J. Math., 27(2):297-323, 2001.

[Mag03] Valentino Magnani. A blow-up theorem for regular hypersurfaces on nilpotent groups. Manuscripta Math., 110(1):55-76, 2003.

[Mat95] Pertti Mattila. Geometry of Sets and Measures in Euclidean Spaces: Fractals and rectifiability. Cambridge University Press, 1995.

[Mit85] John Mitchell. On Carnot-Carathéodory metrics. Journal of Differential Geometry, 21:35-45, 1985.

[Mon02] Richard Montgomery. A tour of subriemannian geometries, their geodesics and applications. Mathematical Surveys and Monographs, 91. American Mathematical Society, 2002.

[MM02] Roberto Monti and Daniele Morbidelli Trace theorems for vector fields. Math. Z., 239(4):747-776, 2002.

[Pan89] Pierre Pansu. Métriques de Carnot-Carathéodory et quasiisométries des espaces symétriques de rang un. Annals of Mathematics (2), 129(1):1-60, 1989.

[Pau98] Scott D. Pauls. The large scale geometry of nilpotent Lie groups. Communications in Analysis and Geometry, 9(5):951-982, 2001.

[Sim83] Leon Simon. Lectures on geometric measure theory. Australian National University, Centre for Mathematical Analysis, Canberra, 1983.

[Var81] A. N. Varchenko. Obstructions to local equivalence of distributions. Mat. Zametki, 29(6):939-947, 957, 1981.

[VU96] S. K. Vodopyanov and A. D. Ukhlov. Approximately differentiable transformations and the change of variables on nilpotent groups. Sibirsk. Mat. Zh., 37(1):70-89, ii, 1996.

[Whi98] Brian White. A new proof of Federer's structure theorem for $k$-dimensional subsets of $\mathbf{R}^{N} . J$. Amer. Math. Soc., 11(3):693-701, 1998.

Dartmouth College, Hanover, NH 03755

E-mail address: scott.pauls@dartmouth.edu 